Preprints of the

Max Planck Institute for

Research on Collective Goods

Bonn 2014/13

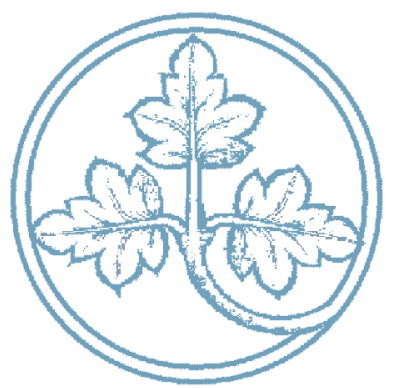

Die normative Ko-Evolution von Marktwirtschaft und Demokratie

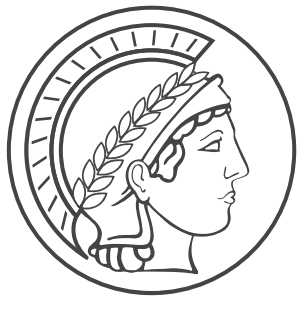




\section{Die normative Ko-Evolution von Marktwirtschaft und Demokratie}

Carl Christian von Weizsäcker

September 2014 


\begin{abstract}
Carl Christian von Weizsäcker
Max-Planck-Institut zur Erforschung von Gemeinschaftsgütern
\end{abstract}

Bonn

September 2014

Die normative Ko-Evolution von Marktwirtschaft und Demokratie ${ }^{1}$

Abstract: Die deliberative Demokratie setzt die Marktwirtschaft als ein System des wirtschaftlichen Wettbwerbs voraus. Dies kann anhand des Popperschen Konzepts einer Offenen Gesellschaft mit Piecemeal Engineering nachgewiesen werden. Denn die Kohärenz einer Offenen Gesellschaft setzt bei endogen beeinflussten Präferenzen voraus, dass diese Präferenzen adaptiv sind. Ohne Marktwirtschaft würden adaptive Präferenzen jedoch zu gesellschaftlicher Erstarrung führen. - Andererseits setzt die Legitimierung der Marktwirtschaft auch die deliberative Demokratie voraus. Denn die interpersonellen Einflüsse auf Präferenzen und Werte bedürfen zu ihrer Legitimierung des Wettbwerbs dieser Einflüsse, den es nur im Rahmen einer demokratisch strukturierten Öffentlichkeit gibt. Die Kohärenz eines solchen Systems wird gestützt durch die Tatsache, dass man interpersonelle Präferenzeinflüsse oft in Analogie zu adaptiven Präferenzen sehen kann. Das gilt insbesondere für das universelle Phänomen der Nachahmung.

JEL Classification: D03, D11, D60, D61, P10, Z10

\footnotetext{
${ }^{1}$ Ich danke Theodor Baums, Beat Blankart, Christoph Engel, Armin Falk, Dominik Grafenhofer, Helena Helfer, Carsten Hermann—Pillath, Karen Horn, Gebhard Kirchgäßner, Christian Müller, Niels Petersen und Jan Schnellenbach für schriftliche oder mündliche Anregungen bei der Vorbereitung dieses Textes.
} 


\section{Gliederung}

A Einleitung und Zusammenfassung

B Gemeinsame Voraussetzungen: Rechtsstaat, Fortschritt und staatliches Gewaltmonopol

C Der Begriff der Präferenzen und der Begriff der Freiheit

D Adaptive Präferenzen: Zur Kohärenz der Prinzipien der Offenen Gesellschaft

E Demokratie setzt Marktwirtschaft voraus. Teil 1

F Demokratie setzt Marktwirtschaft voraus. Teil 2

G Marktwirtschaft wird durch Demokratie legitimiert. Teil 1

H Marktwirtschaft wird durch Demokratie legitimiert. Teil 2

I Schlussbetrachtung

Anhang 1: Theoreme 1 und 2

Anhang 2. Ein einfaches Beispiel für die formale Äquivalenz von nachahmenden und adaptiven Präferenzen.

Literatur 


\section{A Einleitung und Zusammenfassung}

Der Blick auf das Gemeinwohl ist der Blick auf das Ganze der Gesellschaft. Diese sehr alte Erkenntnis der Sozialphilosophie ist auch bei den Vordenkern einer guten Wirtschaftsordnung nicht vergessen worden. Die Arbeiten von Eucken, Rüstow, Röpke, Hayek und anderen belegen dies. Indessen ist in der engeren Theorie der Wirtschaftssysteme eine Abkoppelung zwischen dem gesamtstaatlichen Gemeinwohl und dem "wirtschaftlichen" Gemeinwohl zu beobachten. Diese Abkoppelung gehörte im Kern schon zu den Geburtswehen", die mit der Entstehung der Ökonomik als Wissenschaft verbunden waren. Die Kritik an der liberalen politischen Ökonomie basiert nicht zuletzt darauf, dass diese Isolierung des "rein Wirtschaftlichen" nicht zulässig sei. Sowohl die konservative als auch die sozialistische Kritik an der liberalen Wirtschaftstheorie basiert sehr weitgehend auf der Problematik dieser Abkoppelung.

Indessen gibt es zwei Verteidigungslinien der liberalen Theorie zugunsten der Abkoppelung - von der Behauptung der leichteren intellektuellen Durchdringbarkeit abgesehen. Einmal kann sie auf den fundamentalen Erfolg des liberalen Wirtschaftssystems hinweisen: indem man nach den liberalen Rezepten eine Wirtschafts- und Staatsordnung errichtete, in der diese Abkoppelung in die Praxis umgesetzt wurde, gelang die "Große Transformation" (Karl Polanyi) ${ }^{3}$, die den materiellen Lebensstandard der Bevölkerung um eine Größenordnung und die Lebenserwartung um den Faktor Zwei bis Drei erhöhte. Wenn aber die Eigendynamik, also die Entpolitisierung des Wirtschaftssektors der Gesellschaft diese Erfolge zeitigt, sollte es legitim sein, die Erscheinungen des rein wirtschaftlichen Gemeinwohls auch abgekoppelt von den übrigen Gemeinwohlaspekten zu analysieren.

Die zweite Verteidigungslinie einer analytischen Abkoppelung des wirtschaftlichen Gemeinwohls beruht ebenfalls auf einer historischen Erfahrung, nämlich der des Totalitarismus des Zwanzigsten Jahrhunderts. Die

\footnotetext{
${ }^{2}$ Im Jahre 1749 erschien der zweite Band von Montesquieus "L'Esprit des Lois", beginnend mit dem vierten Teil des Gesamtwerks. Dieser Teil war dem "Commerce" gewidmet. Montesquieu eröffnet nur diesen Teil mit einer Anrufung der Musen, die er bittet, ihm zu helfen, den schwierigen Stoff zu meistern. Montesquieus Anschauungen zur Wirtschaftspolitik fanden Gnade vor John Maynard Keynes. Im Vorwort zur französischen Ausgabe der General Theory schreibt er: "Montesquieu was the real French equivalent of Adam Smith. The greatest of your economists, head and shoulders above the physiocrats in penetration, clear-headedness and good sense (which are the qualities an economist should have)." Keynes (1939)

${ }^{3}$ Polanyi (1944)
} 
Bewegungen, die antraten, das Glück der Menschen auf direktem Wege einer integralen Gemeinwohlorientierung zu erreichen, schufen die Freiheit des Bürgers im (vermeintlichen) Interesse eines Gesamtwohls ab - und sie scheiterten. Karl Popper hat diese Kritik an der Utopie-Orientierung der Politik in seinem Buch über die Offene Gesellschaft am klarsten formuliert ${ }^{4}$, indem er den geistigen Zusammenhang zwischen dem utopischen Denken und der totalitären Praxis aufdeckte. Man kann dies auch so ausdrücken: Der Primat der Politik vor den anderen Lebensbereichen (den Mussolini, Stalin und Mao Tse Tung gleichermaßen für ihre Taten reklamierten) hat versagt. In ähnlicher Weise wie Popper hat Hayek in seiner Kritik am "Konstruktivismus" argumentiert ${ }^{5}$.

Die neoklassische Orthodoxie war recht erfolgreich gerade auch bei der normativen Durchdringung der Materie, soweit es das wirtschaftliche Gemeinwohl betrifft. Ich denke hier an die gut ausgebaute "Welfare Economics", nicht zuletzt auch mit ihrer praktischen Umsetzung in der Form der partialanalytischen Kosten-Nutzen-Analyse. Aber sie ist dennoch auf grundlegende Probleme gestoßen. Diese manifestieren sich insbesondere in der berechtigten Kritik am Homo Oeconomicus. Kann die normative ökonomische Theorie den Homo Oeconomicus überwinden? Ich werde in Umrissen zeigen, dass die Antwort "Ja" ist. Zugleich bietet jedoch meine Fortentwicklung dieser normativen Theorie auch die Chance, die genannte Abkopplung des wirtschaftlichen Gemeinwohls vom umfassenden Gemeinwohl in einem Teil zu rechtfertigen und in einem anderen Teil auch wieder zu überwinden. Es entsteht damit eine neue Brücke zur Allgemeinen Staatslehre, wie sie in der Philosophie und in der Rechtslehre betrieben wird. Dieser Beitrag sieht sich daher in der Tradition des Denkens von Montesquieu, eines Denkens, das vom Mainstream der Ökonomik weitgehend vergessen wurde. Er sieht sich zugleich als Teil des Gebiets der Ökonomik, das heute "Public Choice" genannt wird.

Meine Analyse führt mich zu den folgenden zwei Thesen: 1. Die Marktwirtschaft ist Bedingung für eine demokratische Grundordnung des Staates. 2. Die politische Demokratie ist Bedingung für eine legitimierbare und damit letztlich stabile Marktwirtschaft.

\footnotetext{
${ }^{4}$ Popper (1945)

${ }^{5}$ Hayek (1944)
} 
Das Wirtschaftssystem ebenso wie das politische System unterliegen einem immerwährenden Prozess der Evolution. Es gibt somit auch eine Ko-Evolution der beiden Systeme. Um Habermas zu zitieren, gilt dies nicht nur auf der Ebene der "Faktizität", sondern auch auf der normativen Ebene der "Legitimität" oder der "Geltung"6. Diesen Befund bezeichne ich auch als die "normative KoEvolution von Marktwirtschaft und Demokratie. "“7

Ökonomen und Sozialphilosophen haben auch über die faktische - im Gegensatz zur normativen - Ko-Evolution von Marktwirtschaft und Demokratie nachgedacht. So gibt es die sogenannte Hayek-Friedman-These, dass Marktwirtschaft eine Grundvoraussetzung für eine freiheitliche Demokratie sei. Sie ist nicht das Hauptthema dieser Arbeit $^{8}$. Indessen vermute ich, dass eine stabile Symbiose von Marktwirtschaft und Demokratie auch darauf beruht, dass es eine solche Symbiose auch auf der normativen Ebene gibt.

Zusammenfassung des Folgenden: Es geht mir primär um normative Theorie auf individualistischer Grundlage. Vorausgesetzt wird das Gewaltmonopol des Staates, ein funktionierender Rechtsstaat und die Orientierung der "Offenen Gesellschaft" in Sinne Karl Poppers am Prinzip des inkrementellen Fortschritts. Dieser soll festgemacht sein an den Präferenzen der Bürger. Ich zeige: Ein Präferenzensystem mit sich endogen veränderenden Präferenzen (anstelle der fixen Präferenzen des Homo Oeconomicus) kann einen kohärenten Begriff von Fortschritt nur haben, wenn diese Präferenzanpassungen adaptiv sind. Damit ist verbunden eine jeweilige Aufwertung des Status Quo. Würden alle Entscheidungen -nach dem Gedanken eines "Primats der Politik- zentral gefällt, dann führten die demokratischen Mehrheitsentscheidungen wegen dieser jeweiligen Aufwertung des Status Quo in die Stagnation. Nur eine Dezentralisierung der Entscheidungen, sprich, eine wettbwerbliche Marktwirtschaft und ein System der Forschungsfreiheit in der Wissenschaft kann diese Status-Quo-Orientierung der Gesellschaft erfolgreich überwinden. Dezentrale Entscheidungen sind solche, die entlastet sind von der direkten Orientierung am Gemeinwohl. Der institutionelle Rahmen der Gesellschaft soll

\footnotetext{
${ }^{6}$ Habermas (1992)

${ }^{7}$ Um im vorgesehenen quantitativen Rahmen eines Artikels zu bleiben, muss ich hier die Aspekte

"Gerechtigkeit", "Sozialstaat" und "Soziale Marktwirtschaft" ausblenden. Diese Aspekte sollen an anderer Stelle behandelt werden. Gleiches gilt für die ökologischen Aspekte.

${ }^{8}$ Vgl. zur Hayek-Friedman-These die Arbeit von Lawson et al (2010).
} 
dafür sorgen, dass eigennützig gefällte dezentrale Entscheidungen zugleich dem Gemeinwohl dienen.

Auf der anderen Seite können die Präferenzen der Bürger als Quelle der gesellschaftlichen Werte nur legitimiert werden, wenn eine deliberative Demokratie herrscht. Denn, nach meinem Vorschlag können Ergebnisse interpersoneller Einflüsse auf Präferenzen nur legitimiert werden, wenn es einen Wettbewerb zwischen den "Beeinflussern" gibt. Dass ein solcher Wettbwerb zumindest bei den Einflüssen auf erwachsene Personen vorherrscht, bedeutet nichts anderes als dass man dem Ziel einer deliberativen Demokratie nahe kommt. Es kann gezeigt werden, dass interpersonelle Einflüsse auf Präferenzen dann zu einem kohärenten Begriff von Fortschritt führen, wenn bei diesen Einflüssen die Verhaltens-Nachahmung anderer Personen vorherrscht.

Das Habermassche Idealbild eines herrschaftsfreien Diskurses unter dem "eigentümlich zwanglosen Zwang des besseren Arguments" kann in reiner Form nie verwirklicht werden, da ihm institutionell letztlich das Einstimmigkeitsprinzip zugrunde liegt. Dieses aber würde in einer Gesellschaft mit einem Gewaltmonopol des Staates und rechtsstaatlichen Prozeduren in die Stagnation führen, da die Gegner einer jeweils vorgeschlagenen Abweichung vom Status Quo über ein Vetorecht verfügen würden. Insofern kann eine auf Fortschritt ausgerichtete Gesellschaft auf das Prinzip der Mehrheitsentscheidung nicht verzichten. Mehrheitsentscheidung bedeutet damit aber immer auch Ausübung von Herrschaft einer jeweiligen Mehrheit über eine jeweilige Minderheit. Dem Missbrauch von Mehrheitsentscheidungen muss in der Verfassung durch die Etablierung von Grundrechten gesteuert werden. Diese, insbesondere die Meinungs-, Presseund Versammlungsfreiheit und die Freiheit der Berufsausübung, sind dann auch ein Schutz des sanktionsschwachen freien Diskurses gegen die Verfestigung von Mehrheitsmeinungen ${ }^{9}$.

\footnotetext{
${ }^{9}$ Es besteht eine eigentümliche formale Ähnlichkeit zwischen der Ebene des gesellschaftlichen Diskurses und der Ebene des wettbwerblichen Marktgeschehens. Das Idealbild des Habermas-schen herrschaftsfreien Diskurses entspricht in diesem formalen Vergleich dem Idealbild der Euckenschen vollständigen Konkurrenz, in der die Marktteilnehmer keine Preissetzungsmacht besitzen. Hier wird dieses Idealbild durch den Schumpeterschen Innovator gestört, der durch seine Innovation immer wieder Preissetzungsmacht in den Markt einführt- aber dadurch zum wirtschaftlichen Fortschritt beiträgt. Dort wird das Idealbild des herrschaftsfreien Diskurses durch die jeweiligen Mehrheitsbeschlüsse gestört, die natürlich
} 


\section{B Gemeinsame Voraussetzungen: Rechtsstaat, Fortschritt und staatliches}

\section{Gewaltmonopol}

Es gibt zahlreiche Varianten der Demokratie. Die US-amerikanische Demokratie ist anders als die deutsche, die wiederum anders ist als die französische oder die schweizerische oder die britische. Es gibt auch zahlreiche Varianten der Marktwirtschaft. Die Erhardsche "Soziale Marktwirtschaft" entspricht einem anderen Wirtschaftsstil als dem des US-amerikanischen Kapitalismus, oder dem des japanischen Systems. Diese unterschiedlichen Varianten müssen aus der Sicht einer allgemeinen Systemtheorie oder einer allgemeinen Ordnungstheorie mit Ambivalenz gesehen werden. Einerseits mögen theoretische Einsichten Anlass zur Kritik an einzelnen oder auch allen konkret vorgefundenen Erscheinungsformen von Demokratie oder Marktwirtschaft geben. Andererseits kann man die Varianten auch als Beispiele eines großen Spiels von "Versuch und Irrtum" verstehen. Diese zweite Betrachtungsweise entspricht der Popperschen Philosophie der "Offenen Gesellschaft", die ja durch "Piecemeal Engineering" und "Trial and Error" ausgezeichnet ist und einen Gegenentwurf zu gesellschaftspolitischen Gesamtkonzeptionen darstellt, auf die das Hayeksche Verdikt einer "Anmaßung von Wissen" zutrifft $^{10}$.

Diesem Ansatz eines großen Spiels von "Versuch und Irrtum" entspricht auch mein hier entwickelter Entwurf. Damit ist Kritik an einzelnen Erscheinungsformen von Demokratie oder Marktwirtschaft möglich und nützlich. Aber diese Kritik ist "aufgehoben" in dem Gesamtverständnis, dass man aus Fehlern und Fehlentwicklungen lernen kann und dass man insofern einem kritisierten Missstand auch etwas Positives abgewinnen kann: man lernt daraus, wie man es nicht machen sollte ${ }^{11}$. Und es ist ja ex ante nicht klar, ob eine bestimmte künftige Entwicklung sich zum Guten oder Schlechten auswirkt.

\footnotetext{
Herrschaftsausübung bedeuten - jedoch dafür sorgen, dass die Vetomacht der Gegner einer Veränderung des Status Quo gebrochen wird. Im politischen Bereich muss die Herrschaft der Mehrheit durch verfassungsrechtliche Schranken, insbesondere Grundrechte und rechststaatliche Prozeduren begrenzt werden. Im wirtschaftlichen Bereich muss die potentielle Marktmacht des Innovators durch nachstoßenden Preiswettbwerb begrenzt werden. Auf beiden Ebenen geht es damit um den optimalen Kompromiss zwischen einem Idealbild, das aber status-quo-verhaftet ist, und einem macht-generierenden "Störfaktor", der zu Fortschritt führt, aber eben auch eingehegt sein muss.

${ }^{10}$ Hayek (1973)

${ }^{11}$ Die hier vorgelegte Analyse gewinnt - so meine ich - an Überzeugungskraft durch die historische Erfahrung des Scheiterns der Zentralverwaltungswirtschaft. Dieses Scheitern wurde von Hayek vorausgesagt und ist von Kornai, der es selbst durchlitten hat, ex post hervorragend erklärt worden. Vgl. Kornai (1992)
} 
Der Popperschen Rezeptur eines "Piecemeal Engineering" entspricht das Anerkenntnis eines vorgefundenen Status Quo. Wird im Rahmen einer Philosophie des "Trial and Error" über ein neues soziales Experiment nachgedacht, so gibt es immer auch die Möglichkeit, bei dem jeweiligen Status Quo zu verharren, somit also auf dieses soziale Experiment zu verzichten. Wir sprechen hier auch von der "Default Option": wird bezüglich eines hypothetischen Experiments nichts entschieden, so bleibt es beim Status Quo. Oder anders ausgedrückt: jede Abweichung vom Status Quo ist verbunden mit einer bewussten und expliziten Entscheidung, etwas zu verändern.

Die beiden in der Einleitung formulierten Thesen machen nur Sinn, wenn die Begriffe "Demokratie" und "Marktwirtschaft" nicht völlig schwammig sind, sodass man unter sie "alles" subsumieren könnte. Es ist somit erforderlich, einen Kernbestand zu formulieren, der demokratische Systeme von anderen politischen Systemen und der marktwirtschaftliche Systeme von anderen Wirtschaftssystemen abgrenzt.

Beiden Begriffen soll gemeinsam sein, dass es ein Gewaltmonopol des Staates gibt. Die Demokratie beruht auf dem Prinzip, dass eine Mehrheit von wahlberechtigten Bürgern frei darüber entscheidet, ob im staatlichen Bereich eine Änderung am Status Quo vorgenommen wird. Dazu gehört aber auch, dass die getroffene Mehrheitsentscheidung ohne großen Widerstand durchgesetzt werden kann. Regt sich gewaltsamer Widerstand gegen die legitime Mehrheitsentscheidung, so handelt es sich hierbei um eine gravierende Abweichung vom Demokratieprinzip. Auch das Zustandekommen einer Mehrheitsentscheidung bedarf $\mathrm{zu}$ ihrer Legitimierung, dass sich alle wahlberechtigten Bürger frei, also insbesondere ohne die Androhung von Gewalt durch Dritte entscheiden können. Daher setzt eine funktionierende Demokratie voraus, dass es ein Monopol des Staates auf legitime Gewaltausübung gibt und dass der Staat auch stark genug ist, illegitime Gewaltausübung durch andere weitgehend zu unterbinden.

Gleiches gilt aber auch für die Marktwirtschaft. Ein wesentlicher Bestandteil ist der freiwillig vorgenommene Tausch. Dieser kann sich in einer Gesellschaft nur entfalten, wenn es keine Gewaltandrohung zwischen den Bürgern gibt. Auch dies führt zum Gewaltmonopol des Staates. Zudem erfordert ein Marktsystem gesicherte Eigentumsrechte. Diese setzen ein funktionsfähiges Rechtssystem 
voraus, mit dessen Hilfe aus Eigentum entstandene oder vertraglich entstandene Ansprüche auf dem Gerichtswege und nicht mittels Ausübung privater Gewalt durchgesetzt werden können. Auch hierfür ist das Gewaltmonopol des Staates Voraussetzung.

Zugleich ist eine gemeinsame Voraussetzung von Marktwirtschaft und Demokratie, dass der Staat als Rechtsstaat organisiert ist. Dies ist, wie schon festgestellt, Voraussetzung für ein funktionsfähiges System der Handlungs- und Vertragsfreiheit. Der Rechtsstaat ist aber auch Voraussetzung für die Demokratie. Diese gewinnt, wie schon bemerkt, ihre Entscheidungsfähigkeit durch das Mehrheitsprinzip. Aber die staatliche Gewalt muss eingezäunt werden durch das Legalitätsprinzip und durch eine unabhängige Gerichtsbarkeit. Die Montesquieu'sche Gewaltenteilung wird hier somit ebenfalls als Bedingung für Demokratie und Marktwirtschaft vorausgesetzt.

Auf der Basis des staatlichen Monopols legitimer Gewaltausübung ergibt sich eine weitere Gemeinsamkeit von Demokratie und Marktwirtschaft: Abweichungen vom Status Quo auf dem Wege des "Trial and Error" sollten zu Fortschritt und nicht zu Rückschritt führen. Die Einführung eines neuen Produkts im Markt wird aus normativer Perspektive dadurch legitimiert, dass man von ihm im Saldo gesamtgesellschaftlich Nutzen und nicht Schaden erwartet. Eine per Mehrheitsbeschluss beschlossene Gesetzesänderung hat zum Ziel, dass sich damit die Situation gesamtgesellschaftlich gesehen verbessert und nicht verschlechtert. Beide, Marktwirtschaft und Demokratie sind als Kinder der Aufklärung fortschrittsorientiert. Nur damit sind sie auch kompatibel mit dem Popperschen Ansatz einer Offenen Gesellschaft, in der Veränderungen des jeweiligen Status Quo auf dem Wege des "Piecemeal Engineeering", des "Trial and Error" erfolgen.

Ein bedeutsamer Unterschied zwischen Demokratie und Marktwirtschaft ist die Entscheidungsstruktur. In der Demokratie geht es um politische Entscheidungen, die als solche zentral für alle Bürger und im Namen aller Bürger gefällt werden. In der Marktwirtschaft werden die Entscheidungen dezentral gefällt, und sie sind privater Natur. Welche Entscheidungen werden politisch, also zentral, und welche Entscheidungen werden privat, also dezentral, gefällt? Dies ist eine der großen Gestaltungsfragen jeder Gesellschaft. 
Natürlich gibt es auch in der Marktwirtschaft zentralisierende Strukturen, so wie es in der Politik dezentralisierende Strukturen gibt. Im Rahmen eines Unternehmens werden zahlreiche Entscheidungen unternehmens-zentral, also von der Unternehmensspitze gefällt. Im Rahmen des staatlichen Apparats werden Entscheidungen sekundärer Art an einzelne Teile der Verwaltung delegiert; sie betreffen direkt dann meist auch nicht alle Bürger, sondern nur jeweils einen Teil von ihnen, häufig nur einen kleinen Teil. Indessen werden Unternehmen in ihrer Existenz legitimiert durch den Wettbewerb im Markt, also durch ein dezentrales Prinzip. Und dezentrale Entscheidungen im staatlichen Bereich werden legitimiert durch Delegation von Befugnissen der obersten politischen Zentrale.

Zusätzliche Wesensbestandteile von Demokratie einerseits und von Marktwirtschaft andererseits werden im Verlauf der weiteren Argumentation ins Spiel kommen. Zuerst soll in den nächsten zwei Abschnitten der Begriff der Präferenzen erläutert und dann die Logik des Piecemeal Engineering noch etwas genauer betrachtet werden.

\section{$\underline{C \text { Der Begriff der Präferenzen und der Begriff der Freiheit }}$}

Bei einem Roboter oder Computer spricht man - bisher - nicht davon, dass er Präferenzen habe. Er ist ein programmiertes kybernetisches System, das ohne eigenen Willen allein den Kausalgesetzen der Physik gehorcht. In der normativen Theorie der Ökonomik oder anderer Sozialwissenschaften benötigt man jedoch die Vorstellung des freien Willens der handelnden Personen. Die Funktion des Begriffs der Präferenzen ist diese: sie soll das Verhalten von Menschen beschreiben, denen man einen freien Willen unterstellt. Man kann ein bestimmtes Verhalten durch eine Kausalanalyse oder durch Verweis auf die Präferenzen, d.h. die Willensentscheidung der Person erklären. Welchen Zugang zur Erklärung des Verhaltens man wählt, hängt davon ab, welches Erkenntnisprogramm man verfolgt. Wir unterscheiden hier zwischen der positiven und der normativen Theorie. In der positiven Theorie will man Verhalten von Menschen nach dem Kausalprinzip möglichst weitgehend erklären. Hier auf die Präferenzen zu verweisen ist eigentlich nur das Anerkenntnis, dass man das Verhalten kausal (noch) nicht vollständig erklären 
kann $^{12}$. In der normativen Theorie des "normativen Individualismus" will man die bürgerliche Freiheit der Person beschreiben. Hier dient der Erklärungsdualismus von Beschränkungen (z.B. Budgetbeschränkungen) und Präferenzen dazu, das Verhalten einerseits durch "Sachzwänge" (=Beschränkungen) und andererseits durch die freie Entscheidung der Person (Präferenzen) zu erklären. Wo analytisch der Schnitt zwischen Beschränkung und Präferenzen gemacht wird, hängt in der normativen Theorie daher davon $a b$, welche Tatbestände man der wirtschaftspolitischen Verfügbarkeit zugänglich machen will und welche man dem rein privaten Bereich der Bürger überlassen will. Im letzteren Fall wird die Entscheidung des Bürgers als Ausfluss seiner Präferenzen, also seiner persönlichen Freiheit, gesellschaftlich legitimiert.

Ein Beispiel ist die politische Wahl oder Abstimmung in der Demokratie. Sie wird geheim durchgeführt, damit der Wähler keinem sozialen Druck ausgesetzt wird. Die Wahl oder Abstimmung soll dem sanktionsfreien Bereich gesellschaftlichen Handelns zugehören. Die Stimme des Wählers zählt und ist damit als Ausdruck seiner Präferenzen legitimiert, gleichgültig, wen er wählt oder wie er abstimmt. Die Ursachen, die zu seiner Wahlentscheidung geführt haben, sind völlig irrelevant dafür, ob seine Stimme zählt oder nicht. Demgegenüber sind die empirische Wahlforschung und letztlich natürlich auch die zur Wahl stehenden Parteien sehr wohl an den Ursachen dieser Wahlentscheidung interessiert. Aber das ändert nichts daran, dass diese Ursachen bei der Legitimierung der Stimmabgabe keine Rolle spielen.

Der Wahlforscher entwickelt ein Kausalmodell, z.B. in der Form einer empirisch gestützten Regression, wo die erklärenden Variablen als Ursache und die zu erklärende Variable als Wirkung interpretiert werden. So stellt er die Verbindung zwischen Wahlursache und Wahlergebnis her. Für das Funktionieren der Demokratie ist es jedoch entscheidend, dass gerade ein Schnitt gemacht wird zwischen Ursache und Wirkung, soweit es die Legitimität der Wahlentscheidung betrifft. Der kognitive Prozess, der zu einer bestimmten Wahlentscheidung führt, ist aus der Sicht der normativen Legitimierung der Stimmabgabe reine Privatsache des Wählers. Nur durch diesen Schnitt ist es der

\footnotetext{
${ }^{12}$ Das ist der Clou des berühmten Aufsatzes von Stigler und Becker (1977) und seines Titels: De gustibus non est disputandum.
} 
Fall, dass am Wahlabend das Wahlergebnis feststeht und die Politik des Landes nunmehr mit diesem endgültigen Wahlergebnis weiter arbeiten kann.

Analog dazu kann man das Konsumverhalten der Bürger einerseits als Ausdruck der Präferenzen der Bürger ansehen und andererseits durch die "Sachzwänge" erklären. In einem bestimmten gesetzgeberischen Status Quo der betrachteten Popperschen "Offenen Gesellschaft" verfügt der Bürger über bestimmte Freiheitsräume, die von seiner persönlichen Lage, insbesondere auch von seiner wirtschaftlichen Lage, aber auch von der allgemeinen Gesetzeslage abhängen. Im Rahmen dieses Freiheitsraums modelliert man seine Entscheidung als Ausfluss seiner Präferenzen. Wenn nun aus pragmatischen Gründen Unzufriedenheit mit dem Status Quo besteht, dann machen sich diese (Verteilungsfragen hier ausgeklammert) in der Regel daran fest, dass man glaubt, Probleme zu beobachten, die einer höheren Wohlfahrt zahlreicher Bürger entgegenstehen. Dann müssen die entsprechenden Problembereiche explizit modelliert werden. Sie werden damit aus dem Erklärungsschema "Präferenzen" herausgehoben und einer eigentlichen Kausalanalyse zugeführt. Nach dieser Kausalanalyse werden vielleicht die Gesetze geändert, was zur Folge hat, dass nunmehr auch die Beschränkungen, denen das Handeln der Bürger unterliegt, sich ändern.

Ein Beispiel: Haustürgeschäfte. Solange hier im Rahmen der allgemeinen Handlungsfreiheit der Bürger noch kein Problem gesehen wird, werden Entscheidungen von Bürgern und Bürgerinnen, welche Waren sie an der Haustür von Verkäufern kaufen, einfach als Ausdruck der Präferenzen der Käufer aufgefasst. Entsteht nun ein Problembewusstsein, indem man beobachtet, dass viele Bürger und Bürgerinnen später diese Käufe an der Haustür bereuen, dann analysiert man die Situation genauer und kommt vielleicht zum Schluss, dass es ein nicht abdingbares Recht des Käufers geben sollte, an der Haustür gekaufte Waren innerhalb einer bestimmten Frist gegen Rückerstattung des Kaufpreises zurück zu geben. Damit wird die Vertragsfreiheit der Bürger seitens des Gesetzgebers bewusst eingeschränkt, indem der Kauf an der Haustür im Moment der Entscheidung nur noch ein vorläufiger Kauf und Verkauf sein kann. Dies mag die Attraktivität der Haustürgeschäfte für die Verkäufer verringern, sodass die Anbahnungen von Haustürgeschäften sich vermindern; dies vielleicht zum Nutzen vieler Bürger, zugleich aber vielleicht zum Schaden einiger Bürger, denen nunmehr auch 
weniger Waren an der Haustür angeboten werden, deren Kauf sie nicht bereut hätten.

Diesem Beispiel zunehmender Regulierung füge ich ein Beispiel verminderter Regulierung an: die Legalisierung der Homosexualität. Solange die Homosexualität unter Strafe gestellt war, unterlag dieser Norm eine Kausaltheorie der Folgen der Homosexualität. Mit ihrer Legalisierung werden sexuelle Praktiken insoweit Privatsache und somit einfach Ausdruck der Präferenzen der Bürger. Sie sind damit gesellschaftlich legitimiert.

Im Umgang mit dem Präferenzbegriff sehen wir somit eine gewisse Parallele zwischen der positiven Theorie und der normativen Theorie, soweit wir letztere im Rahmen des Konzepts der "Offenen Gesellschaft" behandeln. In der positiven Theorie repräsentiert der Begriff der Präferenzen den Rest unerforschter Kausalität in der Verhaltensanalyse des Wirtschaftssubjekts. In der normativen Theorie repräsentiert der Begriff der Präferenzen den gegenwärtig nicht politisch problematisierten Handlungsspielraum des Bürgers. Diese Präferenzen sind aber zugleich Maßstab für die Bewertung von Konsummöglichkeiten, die die Bürger haben. Sie sind der Bewertungsmaßstab für den gesellschaftlich gegenwärtig nicht hinterfragten Teil der bürgerlichen Privatautonomie.

Dieses Vorgehen führt zugleich zur Möglichkeit allgemeiner Theorien über die Wohlfahrtseffekte von Märkten oder anderer Institutionen, indem man im Rahmen einer Partialanalyse Konsumenten- und Produzentenrenten definieren kann. Man kommt dann vielleicht zu dem Ergebnis, dass im Allgemeinen mehr Wettbewerb gut für die Wohlfahrt ist. Eine derartige Analyse mag darüber hinaus für Teilgruppen von Märkten wieder infrage gestellt werden, weil man beispielsweise auf Informationsmängel bei den Nachfragern hinweist. Diese Relativierungen der allgemeinen Theorie werden häufig auch mit gesetzgeberischen Reformvorstellungen verbunden sein.

In der Offenen Gesellschaft gibt es keine endgültigen Wahrheiten und Bewertungen. Indem Probleme neu erkannt werden, und damit Teile der bürgerlichen Privatautonomie hinterfragt werden, schrumpft der Erklärungsanteil der Präferenzen an dem Verhalten der Bürger zugunsten einer genaueren Betrachtungsweise des "Sozialingenieurs" dessen, was die "eigentliche" Wohlfahrt des Bürgers sei. Indem andererseits, zum Beispiel 
durch technischen Fortschritt oder durch veränderte Werte, neue Handlungsalternativen der Bürger entstehen, erhöht sich wieder der Erklärungsanteil der Präferenzen an dem Verhalten der Bürger.

Der Begriff der Präferenzen als Wertmaßstab setzt damit von vorneherein voraus, dass diese Präferenzen sich im Zeitverlauf und in Abhängigkeit des gesellschaftlichen Geschehens ändern. Die fixen Präferenzen des Homo Oeconomicus haben in diesem Ansatz keinen Platz. Zugleich öffnet sich hier einerseits die Möglichkeit, im sozialkritischen Sinne nach versteckten Zwängen zu suchen, die sich hinter der scheinbar freien Entscheidung des Bürgers verbergen; dies allerdings im Sinne des "Piecemeal Engineering" konkret und lokal und nicht im Sinne einer pauschalen Propagierung einer "Kulturrevolution". Andererseits kann dieser Ansatz auch im interventionskritischen Sinne verwendet werden, indem der Begriff der bürgerlichen Freiheit ernst genommen wird und vorgeschlagene staatliche Interventionen im Geiste des "Sozialingenieurs" auf ihre möglicherweise problematischen Modellannahmen abgeklopft werden: dieser Präferenzbegriff impliziert, dass es der Interventionist ist, der die "Beweispflicht" hat.

\section{Adaptive Präferenzen: Zur Kohärenz der Prinzipien der Offenen Gesellschaft}

Die einheitliche Unterstellung aller Politik unter ein weit entferntes und daher recht abstrakt definiertes Ziel, mithin unter eine Utopie, war nach Popper die geistige Basis totalitärer Regimes. Ihr stellte er das anti-totalitäre Konzept der "Offenen Gesellschaft" gegenüber. Hier gehe ich auf die Details dieses Konzepts nicht ein. Ich konzentriere mich auf die Frage, nach welchen Zielen die staatliche Politik einer solchen Offenen Gesellschaft streben sollte. Popper gab die oben schon genannte Antwort des "Piecemeal Engineering", des Voranschreitens nach dem Prinzip von "Trial end Error", von "Versuch und Irrtum". Man spricht hier auch von "Inkrementalismus". Begründet wird dieser Vorschlag damit, dass es - so wie nach Popper auch in der Wissenschaft - in der Politik keine endgültigen Wahrheiten und Werte gibt. Man weiß in der Politik nicht im Voraus, was die Konsequenzen politischen Handelns sind. Und man weiß in der Politik nicht im Voraus, welche Ziele man in der Zukunft anstreben will. 
Ohne dass dies in dieser Form bei Popper ausformuliert wäre, folgt doch aus seinem Ansatz, dass es einen jeweiligen Status Quo gibt, der der Ausgangspunkt für den nächsten Schritt des Piecemeal Engineering ist. Da eine bewusste Veränderung immer Folgen hat, die nicht alle sofort, sondern im Verlauf der Zeit auftreten, werden sich die Folgen von Veränderungsentscheidungen zeitlich überlappen. Der Status Quo ist somit nicht aufzufassen als ein stationärer Zustand; er hat aufgrund von früheren verschiedenen Veränderungsentscheidungen, die sich auch heute und in Zukunft noch auswirken, selbst das Erscheinungsbild einer mehr oder weniger großen Dynamik. Eine weitere Veränderung soll hier "Projekt" genannt werden. Die Entscheidung, ob ein bestimmtes Projekt in Angriff genommen wird, fällt somit unter Berücksichtigung der zu erwartenden Dynamik des Gesamtsystems, wobei quasi "komparativ dynamisch" die zu erwartende Welt mit und ohne die Implementierung des Projekts verglichen wird.

Beispiele für derartige Projekte in der modernen entwickelten Gesellschaft mögen sein: 1. Die Veränderung eines Gesetzes durch die Legislative; 2. Die veränderte Rechtsprechung der obersten Gerichte bezüglich der existierenden Gesetze oder bezüglich der Verfassung; 3. Der Bau einer Brücke über einen Fluss oder ein sonstiges neues Infrastrukturprojekt; 4. Die Einführung eines neuen Produkts auf dem Markt durch ein Unternehmen. Bei all diesen Beispielen wird klar, dass deren jeweilige Wirkungen sich über die Zeit erstrecken und sich daher mit den Wirkungen anderer "Projekte" zeitlich überlappen.

Die Einstellungen und Bewertungen der Güter, die den Bürgern eines Staates zur Verfügung stehen, ändern sich im Zeitverlauf. Dabei sind diese Bewertungsänderungen durchaus auch endogen bestimmt. Das soll heißen: die Bewertungen hängen von den Erfahrungen ab, die die Menschen in dieser Gesellschaft machen. Zu fragen ist: kann eine solche Praxis des Piecemeal Engineering eine gewisse Kohärenz aufweisen, wenn doch die Bewertungen und Zielsetzungen der Bürger des Landes sich endogen verändern, wenn die Zwecke des Handelns sich im Verlauf dieses Handelns und als Ergebnis dieses Handelns verschieben?

Es sei in Beantwortung dieser Frage vorausgeschickt, dass die Ökonomik in der Form der Kosten-Nutzen-Analyse eine Methodik der Evaluierung von 
"Projekten" anbietet, die unter bestimmten zusätzlichen Bedingungen dann eine hohe Kohärenz aufweist, wenn die Präferenzen der Bürger des Landes fest vorgegeben sind, wenn man also mit dem Homo Oeconomicus rechnet. Über die Details dieser zusätzlichen Bedingungen werde ich an dieser Stelle nichts weiter sagen. Für den Ökonomen ist hier das Stichwort die Theorie des "Second Best". Dahinter steht letztlich (und implizit, d.h. den ökonomischen Forschern meist nicht bewusst) eine Annahme der Systemtheorie von Herbert Simon, nämlich die Annahme, dass auch auf das Wirtschaftsgeschehen Simons These zutrifft: alle komplexen Systeme sind durch das Prinzip der "Near Decomposability" charakterisiert ${ }^{13}$. Auf der Basis dieser Near-Decomposability ist Partialanalyse erst möglich.

Die Frage, der ich mich widmen möchte, ist die nach den Möglichkeiten kohärenten gesellschaftlichen Handelns, wenn sich die Präferenzen und Wertungen der Menschen ständig verschieben - und das endogen verursacht. Dabei bleibe ich dem Ansatz des normativen Individualismus treu: die gesellschaftlich relevanten Wertungen sollen rückführbar sein auf die individuellen Präferenzen und Wertungen der Bürger des Landes.

Setzt man den normativen Individualismus voraus, so liegt es nahe, nach Kohärenzbedingungen gesellschaftlichen Handelns zuerst dort zu suchen, wo die gesellschaftlichen Wertungen und Ziele verankert sind, nämlich beim Individuum, also beim einzelnen Bürger. Ich arbeite also auf der Grundlage der Hypothese: Kohärenz kann gesellschaftliches Handeln in einer Gesellschaft freier Bürger nicht aufweisen, wenn das individuelle Handeln typischerweise nicht kohärent ist $^{14}$. Unter dieser Kohärenzhypothese ist somit Kohärenz individuellen Handelns Voraussetzung für eine gewisse Kohärenz gesellschaftlichen Handelns. Ich verzichte an dieser Stelle bewusst auf eine abschließende Definition des Begriffes "Kohärenz". Dieser muss notwendigerweise ein schwieriger Begriff sein, wenn er kompatibel sein soll mit bestimmten Ausprägungen der "Offenen Gesellschaft", in der es ja wechselnde Mehrheiten und damit einander widersprechende Entscheidungen ebenso geben kann wie das Phänomen der sich endogen ständig ändernden

\footnotetext{
${ }^{13}$ Simon (1963)

${ }^{14}$ Mir ist bewusst, dass es Ansätze gibt, die zeigen sollen, dass "rationale" Ergebnisse im Marktgeschehen auch herauskommen können, wenn man Verhaltensannahmen macht, die üblicherweise nicht dem Rationalmodell entsprechen. Vgl. z.B. Sugden (2004). Allerdings glaube ich nicht, dass ähnliche Ergebnisse für das politische Verhalten gefunden werden können.
} 
individuellen Präferenzen und Wertungen. Indessen ist mit dem Gedanken der Kohärenz derjenige einer gewissen Stabilität der Gesellschaft verbunden. Dass "Offene Gesellschaften" nicht notwendigerweise stabil sind, hat uns die Geschichte gelehrt.

Um Missvertändnisse zu vermeiden, sei hier betont, dass die Kohärenz beim Verhalten des Individuums nicht die Kohärenz der gesellschaftlichen Entscheidungen impliziert. Die Kohärenz individuellen Entscheidens ist damit hilfreich, aber nicht ausreichend für die Kohärenz gesellschaftlichen Entscheidens. Für letztere ist wahrscheinlich neben dem Gewaltmonopol des Staates zusätzlich unter anderem auch Voraussetzung, dass zentral gefällte, also politische Entscheidungen mit einer gewissen Gemächlichkeit erfolgen, die es möglich macht, dass vorab hinreichend genau und ausführlich über diese Entscheidung nachgedacht und debattiert worden ist. Auch deshalb hat der jeweilige politische Status Quo ein so großes Gewicht.

Im Folgenden skizziere ich in groben Zügen die von mir entwickelte Theorie der Kohärenz individuellen Verhaltens. Ich kann zeigen: kohärentes individuelles Handeln setzt voraus, dass die Präferenzen und Wertungen der Bürger "adaptiv" sind. Dabei wird der Begriff der Kohärenz festgemacht am Begriff der Fortschrittssequenz und deren Nicht-Zirkularität.

Wir unterstellen für das Folgende, dass die Präferenzen der Bürger endogen beeinflusst werden. Wir betrachten den Status Quo eines Bürgers, z.B. ausgedrückt durch den Warenkorb $x$, den er innerhalb eines Jahres konsumiert. (Hier sei folgendes angemerkt: das formale Modell betrachtet "Warenkörbe", wie sie in der mikroökonomischen Theorie behandelt werden. Ich bin aber davon überzeugt, dass sich die Theorie auch auf andere Wahlobjekte ausdehnen lässt). Jeder Warenkorb $x$, der tatsächlich konsumiert wird, induziert nun (im einfachsten Modell: mit einem Jahr Verzögerung) bestimmte Präferenzen $\rho(x)$. Der Ausdruck $y(>; \rho(z)) x$ soll nun heißen: Beim vom Warenkorb $z$ induzierten Präferenzen $\rho(z)$ präferiert der Bürger den Warenkorb $y$ gegenüber dem Warenkorb $x$. Der Ausdruck $y(<; \rho(z)) x$ soll heißen: beim vom Warenkorb $z$ induzierten Präferenzen zieht der Bürger den Warenkorb $x$ dem Warenkorb $y$ vor. Wir betrachten nun (im Rahmen des einfachsten Modells) eine Serie von Jahres-Warenkörben $x^{0}, x^{1}, x^{2}, \ldots \ldots x^{T}$ mit der folgenden Eigenschaft $\quad x^{0}\left(<; \rho\left(x^{0}\right)\right) x^{1}\left(<; \rho\left(x^{1}\right)\right) x^{2} \ldots \ldots x^{T-1}(<$ 
; $\left.\rho\left(x^{T-1}\right)\right) x^{T}$. In Worten: die Serie von Jahreswarenkörben habe die Eigenschaft, dass der jeweils nächstfolgende Warenkorb dem vorangegangenen vorgezogen wird, wenn die Präferenzen angesetzt werden, die von dem vorangegangenen Warenkorb induziert worden sind. Eine solche Serie nennen wir eine Fortschrittssequenz. Man beachte, dass es wegen der variablen Präferenzen durchaus sein kann, dass der letzte Warenkorb dem ersten Warenkorb nicht vorgezogen wird, wenn man die Präferenzen zugrunde legt, die vom ersten Warenkorb induziert werden. In diesem Fall kann man davon sprechen, dass der letzte Warenkorb dem ersten Warenkorb nicht direkt, wohl aber indirekt vorgezogen wird, weil der Fortschritt einiger Zwischenetappen bedarf.

Nun können wir unterstellen, dass der Bürger es als rational ansieht, einen "besseren" Warenkorb einem "schlechteren" gemäß den jeweiligen Präferenzen vorzuziehen. Indessen wird er von "wirklicher" Rationalität des Verhaltens nicht sprechen wollen, wenn er auf dem Wege einer Fortschrittssequenz im Kreise herumgeführt wird, also beim Ausgangspunkt wieder ankommt. Um somit die eigenen Präferenzen zur Leitlinie seines Handelns zu machen, muss er voraussetzen, dass derartige Fortschrittssequenzen nicht-zirkulär sind. Die Nichtzirkularität von Fortschrittssequenzen kann somit als eine Kohärenzbedingung für die Politik des "Trial and Error" aufgefasst werden. Und auch die Idee der "indirekten Präferenz" macht natürlich nur dann Sinn, wenn Fortschrittssequenzen nichtzirkulär sind.

Um die Sache plastisch zu machen, erinnere ich an das Märchen vom "Hans im Glück", das in der Märchensammlung der Brüder Grimm zu finden ist. Hans beginnt mit einem Goldklumpen und bewegt sich von diesem aus auf einer Fortschrittssequenz, die inn schließlich zum Habenichts macht. Unterstellen wir, dass er nunmehr wieder einen Goldklumpen als Geschenk annehmen würde, so hätte er sich auf einer zirkulären Fortschrittssequenz bewegt. Wäre Hans im Glück typisch für den Bürger der Offenen Gesellschaft, dann könnte man das Poppersche Modell nicht empfehlen.

Indessen bin ich der Auffassung, dass Hans im Glück für die Bürger einer Offenen Gesellschaft nicht typisch ist. Ein wichtiger Grund für diese Auffassung ist folgende: ich kann im Rahmen eines formalen Modells nachweisen, dass 
eine bestimmte Präferenzstruktur, die ich "adaptive Präferenzen" nenne, hinreichend dafür ist, dass jede Fortschrittssequenz nicht-zirkulär ist. Ferner gilt umgekehrt auch folgendes: ist bei einem Bürger die Eigenschaft gegeben, dass jede Fortschrittssequenz nicht-zirkulär ist, dann sind seine Präferenzen adaptiv. Ich halte die Hypothese für realitätsnah, dass die Art, wie sich die Präferenzen der Bürger verändern, adaptiv ist, dass, mit anderen Worten, die Präferenzen adaptiv sind.

Adaptive Präferenzen sind wie folgt definiert: sie gelten dann, wenn aus $y(>; \rho(x)) x$ auch folgt: $y(>; \rho(y)) x$. Das bedeutet, anders ausgedrückt: die Wahrscheinlichkeit, dass ein beliebig heraus gegriffener Warenkorb $x$ weniger hoch geschätzt wird, als ein vorgegebener Warenkorb $y$ ist dann am größten, wenn man mit Präferenzen arbeitet, die von $y$ selbst induziert sind. In diesem Sinne kann man adaptive Präferenzen als Präferenzkonservatismus bezeichnen. Die Menschen haben eine starke Tendenz, dort zu verweilen, wo sie gerade sind. Das ist nicht zu verwechseln mit der Hypothese fixer Präferenzen, die dem Bilde des Homo Oeconomicus entspricht. Denn die Präferenzen eines Franzosen sind typischerweise andere als die Präferenzen eines Deutschen. Und der typische Franzose hat eine größere Vorliebe für alles "Französische" als der typische Deutsche, der eine größere Vorliebe für alles "Deutsche" hat. Fixe Präferenzen, also die Annahme, dass die Präferenzen vom induzierenden Status Quo nicht beeinflusst werden, sind, formal gesehen, übrigens ein Spezialfall der Hypothese adaptiver Präferenzen.

Im Rahmen der Raumvorgaben für diese Arbeit kann ich nicht im Einzelnen darlegen, weshalb ich die Hypothese "adaptiver Präferenzen" für realistisch halte. Das habe ich ansatzweise in anderen Arbeiten getan ${ }^{15}$. Nur auf einen Sachverhalt will ich in diesem Zusammenhang explizit hinweisen. Wie im vorangegangenen Abschnitt $C$ dargelegt, ist der Schnitt, den wir zwischen der Erklärungsgruppe "Beschränkungen" und der Erklärungsgröße "Präferenzen" machen, gegeben durch den im Rahmen des Status Quo bereitstehenden Freiheitsraum der Bürger. Nun gibt es in diesem gesellschaftlich definierten Freiheitsraum "Zwänge" für den Bürger und Gewohnheiten des Bürgers, die gegenwärtig jedoch die Gesellschaft nicht zu interessieren haben. Die Hypothese adaptiver Präferenzen bedeutet dann in diesem Zusammenhang

\footnotetext{
${ }^{15}$ Hierzu im einzlenen von Weizsäcker(2013)
} 
unter anderem auch, dass diese "versteckten", quasi "rein privaten" Einflussfaktoren auf die Entscheidung des Bürgers den jeweiligen Status Quo seines Konsums gegenüber den Alternativen begünstigen, weil es der Status Quo ist. Die privaten Beschränkungen oder Gewohnheiten haben gemäß dieser Hypothese durchgängig die Eigenschaft, dass es sich um Beschränkungen in den Veränderungsmöglichkeiten handelt, die solange nicht anfallen, als man beim Status Quo verbleibt. "Habit Formation" ist eines der Phänomene der empirischen Konsumforschung, die sich immer wieder bestätigen. Auch das bekannte Phänomen der "Switching Costs" ergibt sich nur, wenn man vom Status quo abweichen will. Analoges gilt für das Informationsproblem: da der Status Quo am besten bekannt ist, entstehen Suchkosten vor allem, wenn man vom Status Quo abweichen will.

Eine weitere Kohärenzbedingung der Offenen Gesellschaft ist folgende. Die "Projekte", die unternommen werden, um vom Status Quo abzuweichen, werden deshalb unternommen, weil man mit den gegenwärtigen Präferenzen und Einsichten der Bürger das Ergebnis des Projekts als eine Verbesserung gegenüber dem Status Quo ansieht. Die Entscheidung für ein Projekt fällt somit, weil man das Projekt mit den Präferenzen "ex-ante" befürwortet. Da nun aber die Präferenzen mit der Implementierung dieses Projekts andere werden, entsteht die Frage, ob das Projekt auch noch mit den "ex-post"Präferenzen gutgeheißen wird. Wäre das nämlich regelmäßig nicht der Fall, dann würde die Gesellschaft dieses inkrementelle Abweichen vom jeweiligen Status Quo auf die Dauer nicht tolerieren. Das aber müsste die Rezeptur des "Piecemeal Engineering" inakzeptabel machen.

Nun stellt sich heraus, dass adaptive Präferenzen der Bürger eine gute Voraussetzung dafür sind, dass Projekte auch mit den "ex-post"-Präferenzen begrüßt werden, sofern sie "ex-ante" befürwortet wurden. Die Analyse dieses Sachverhalts ist nicht ganz einfach und kann deshalb an dieser Stelle nicht dargestellt werden ${ }^{16}$. Umgekehrt ist es sehr wahrscheinlich, dass anti-adaptive Präferenzen dazu führen, dass ein Projekt, das ex-ante befürwortet wurde, expost bereut wird.

Es gibt natürlich viele Gründe, weshalb durchgeführte Projekte im Nachhinein bereut werden. Dazu gehört die Unsicherheit über die Kosten und die

\footnotetext{
${ }^{16}$ Vgl. aber von Weizsäcker(2013) und von Weizsäcker (2014a)
} 
Wirkungen eines Projekts. Um dennoch eine gesellschaftliche Bereitschaft zu stabilisieren, Projekte, Reformen, Innovationen immer wieder zu versuchen, muss die Erfahrung vorausgegangen sein, dass diese Veränderungen, wenn sie denn unternommen werden, nicht regelmäßig im Nachhinein durch induzierte Präferenzänderungen desavouiert werden. In dieser Arbeit konzentriere ich mich auf das Problem, das durch endogen verursachte Präferenzänderungen hervorgerufen wird. Ich diskutiere daher nicht in aller Breite die gesellschaftlichen Entscheidungsprobleme, die schon ohne endogene Präferenzänderungen vorhanden sind. Ich verweise für letzteren Zusammenhang nur auf die interessante Beobachtung von Albert Hirschman, die dieser unter der Bezeichnung des "Principle of the Hiding Hand ${ }^{\text {“17 }}$ diskutiert hat.

In Anhang 1 befindet sich eine formale Darstellung der beiden oben genannten Theoreme: 1. Adaptive Präferenzen implizieren die Nichtzirkularität von Fortschrittssequenzen; 2. Sind alle Fortschrittssequenzen nicht-zirkulär, dann sind die Präferenzen adaptiv.

Die Quintessenz dieses Abschnittes ist damit: Eine Kohärenzvoraussetzung für die "Offene Gesellschaft" ist die, dass die Präferenzen der Bürger adaptiv sind. Die Adaptivität der Präferenzen der Bürger ist auch Bedingung dafür, dass die Vorstellung von "Fortschritt" mit einem logisch konsistenten Inhalt gefüllt werden kann.

\section{$\underline{\text { E Demokratie setzt Marktwirtschaft voraus. Teil } 1^{18}}$}

Wir sind nunmehr in der Lage abzuleiten, dass Demokratie als Staatsform nur funktionsfähig ist, wenn sie im wirtschaftlichen Bereich auf einer Marktwirtschaft aufbauen kann.

Demokratie ist durch das Mehrheitsprinzip nicht erschöpfend definiert. Zwar ist dieses notwendiger Teil eines demokratischen Gemeinwesens. Denn Einstimmigkeit kann für politische Entscheidungen realistischerweise nicht gefordert werden. Bei Einstimmigkeit wären die Beharrungskräfte, die an der Aufrechterhaltung des Status Quo interessiert sind, übermächtig. Denn, wie

\footnotetext{
${ }^{17}$ Hirschman (1967)

${ }^{18}$ Der Grundgedanke dieses Abschnittes E ist schon enthalten in von Weizsäcker (2003)
} 
oben ausgeführt, gibt es immer eine "Default Option", die implizit gewählt wird, solange keine Entscheidung explizit gefällt ist. Diese implizite Entscheidung ist immer die Entscheidung zugunsten des Status Quo. Damit der Status Quo nicht übermächtig wird, bedarf es einer höheren Entscheidungskraft zur Überwindung von Widerständen gegen Veränderung. Unter demokratischen Verhältnissen muss allerdings zumindest eine Mehrheit für eine Abweichung vom Status Quo gefunden werden, soweit es sich um politische, also zentral gefällte Entscheidungen handelt. Für bestimmte Entscheidungen mögen sogar "qualifizierte" Mehrheiten festgelegt worden sein.

Aber zur Demokratie gehört auch eine Öffentlichkeit, ein öffentlicher Diskurs. Es ist ja nicht zuletzt die öffentliche Diskussion, die es auch ermöglicht, dass sich Meinungen ändern, dass es nicht zu einer Zementierung der Macht einer einmal zustande gekommenen Mehrheit kommt. Man spricht hier auch vom Ideal der "deliberativen Demokratie". In ihr gehen den Gesetzesänderungen jeweils umfassende öffentliche Diskussionen voraus. Habermas hat das Ideal eines herrschaftsfreien Diskurses formuliert. In ihm sollen die Diskursteilnehmer gleichberechtigt sein und damit nicht durch Machtausübung anderer Teilnehmer in ihrer Argumentation beeinträchtigt werden. Es soll damit keinen äußeren Zwang in der Debatte geben - außer eben den "eigentümlich zwanglosen Zwang des besseren Arguments". Hier soll sich somit auf diese Weise das durchsetzen, was Habermas die "lebenspraktische Vernunft" im Gegensatz zur "instrumentellen Vernunft" nennt; letztere verstanden als die Vernunft zielorientierten Handelns ${ }^{19}$; erstere zusätzlich verstanden als kommunikative Vernunft, die auch die eigenen Zielsetzungen relativiert. Dieser herrschaftsfreie Diskurs ist damit gerade auch ein Diskurs über die Sinnhaftigkeit und Legitimität der jeweils eigenen Zielsetzungen.

Dem gegenüber gilt im Bereich der Wirtschaft, als Teil dessen, was Habermas den Bereich der instrumentellen Vernunft nennt, sehr wohl ein Zwang, und zwar der Zwang des Wettbewerbs. Dieser Wettbewerb ist charakterisiert durch ein System wirkungskräftiger Sanktionen: wer kostengünstig, also ressourcenschonend produziert, kann im Wettbewerb bestehen, weil er zu niedrigen Preisen anbieten kann. Wer zu kostenträchtig produziert, weil er

\footnotetext{
${ }^{19}$ Habermas (1981)
} 
Ressourcen verschwendet, geht unter. Wer gute Qualität zu annehmbaren Preisen kostendeckend liefern kann, macht Gewinn und besteht im Wettbewerb. Wer keine angemessene Qualität zu annehmbaren Preisen liefern kann, dem laufen die Kunden weg, und er geht unter.

So gibt es einen sanktionsstarken Bereich des gesellschaftlichen Lebens, zu dem die arbeitsteilig und wettbewerblich produzierende Wirtschaft gehört; und es gibt im Idealfall einen sanktionsschwachen Bereich der Öffentlichkeit, des öffentlichen Diskurses, in dem das eigene Sprechen und damit Handeln gerade nicht sanktioniert wird, weder negativ noch positiv.

Damit beide Bereiche, der sanktionsstarke und der sanktionsschwache, in derselben Gesellschaft koexistieren können, müssen sie von einander getrennt sein. Somit dominiert in der Idealform der funktionierenden Demokratie und der funktionierenden, also wohlstandsorientierten Wirtschaft ein Trennsystem: die Wirtschaft ist entpolitisiert. Eine entpolitisierte Wirtschaft ist jedoch der Inbegriff der Marktwirtschaft. Eine politisierte Wirtschaft wäre eine, in der man entweder weitestgehend auf Sanktionen verzichtet oder eine, in der auch der öffentliche Raum von starken Sanktionen durchsetzt ist. Im letzteren Fall wäre man weit entfernt vom sanktionsfreien öffentlichen Diskurs und damit weit entfernt von einer deliberativen Demokratie. Die jeweils herrschende Mehrheit würde ihre Macht über die Wirtschaft dazu benutzen, oppositionelle Kritik zu unterbinden und so ihre Machtposition zu perpetuieren. Im ersten Fall einer sanktionsschwachen Wirtschaft nimmt man die Unternehmen aus dem Wettbewerbszwang heraus mit der Folge, dass die Wirtschaft nicht mehr in der Lage ist, sich flexibel anzupassen und Wohlstand zu generieren. Unternehmen ohne Wettbewerb erstarren. In ihnen sind die innerbetrieblichen Widerstände gegen Veränderung übermächtig.

In diesem Sinne haben wir gezeigt, dass die Marktwirtschaft die Voraussetzung für eine funktionierende deliberative Demokratie ist, sofern man auf das Ziel des Wohlstands nicht verzichten will. Natürlich ist das hier formulierte Trennsystem in seiner Idealform nirgendwo auf der Erde verwirklicht. Wirtschaftliche Interessengruppen, große internationale Konzerne haben immer Zugang zur Politik und können sie gemäß ihren Interessen beeinflussen. Dieser Einfluss rührt schon alleine daher, dass dem politischen Personal in der Regel der wirtschaftliche Sachverstand abgeht. Denn in der modernen 
Demokratie qualifiziert man sich zum Politiker, wie zu jedem anderen Beruf, gerade dadurch, dass man sich schon mit jungen Jahren auf das Geschäft des Karrieremachens in der Politik spezialisiert. Das lässt wenig Zeit zum Erwerb von Sachverstand in wirtschafltichen Dingen. Die wettbewerbliche Marktwirtschaft ist somit immer verbesserungsfähig, auch gerade im Hinblick auf die Interdependenz zwischen wirtschaftlichen Interessen und der Politik. Indessen ist ein immer wieder anzumahnendes Distanzgebot zwischen wettbewerblicher Wirtschaft und Politik gerade Ausfluss des Trennprinzips zwischen dem sanktionsstarken Wirtschaftsbereich und dem idealerweise sanktionsschwachen Bereich der Öffentlichkeit.

\section{F Demokratie setzt Marktwirtschaft voraus. Teil 2}

Aber wir können noch einen Schritt weiter gehen, indem wir nun die Kohärenzerfordernisse der Offenen Gesellschaft beachten, die wir im vorletzten Abschnitt abgeleitet haben. Wir können im Sinne der Kohärenz des Popperschen Konzepts voraussetzen, dass die Präferenzen adaptiv und damit status- quo- orientiert sind. Nur in einer Gesellschaft konservativer Bürger kann ein anti-totalitäres Konzept des Piecemeal-Engineering funktionieren. Zwar ist der Homo Oeconomicus ein Spezialfall adaptiver Präferenzen. Indessen liegt er an der Grenze zwischen adaptiven und anti-adaptiven Präferenzen ${ }^{20}$ - und diese "gefährliche" Nähe des Homo Oeconomicus zu Hans im Glück (als dem Prototypen anti-adaptiver Präferenzen) führt dazu, dass die Menschen stark adaptiv in ihren Präferenzen sind. Es gibt starke quasi "soziobiologische" Argumente für stark adaptive Präferenzen, da in einer Welt hoher Unsicherheit eine zu starke Tendenz, dem überschaubaren, daher noch relativ "sicheren" Status Quo zu entfliehen, für das einzelne Individuum die Gefahr des Untergangs massiv erhöht. Die Menschen hängen stark am Status Quo. Sie mögen mit diesem unzufrieden sein; jedoch sind sie nicht leicht zu bewegen, von ihm abzuweichen.

Diese Analyse schließt nicht aus, dass die Menschen massenweise auf Demagogen hereinfallen. Im Gegenteil: gerade die konservative

\footnotetext{
${ }^{20}$ Anti-adaptive Präferenzen sind wie folgt definiert: Gilt $y(<; \rho(x)) x$, dann gilt auch $y(<; \rho(y)) y$. Fixe Präferenzen sind ein Spezialfall sowohl von adaptiven als auch von anti-adaptiven Präferenzen. Sie sind aber auch der einzige derartige Spezialfall.
} 
Grundeinstellung aufgrund der adaptiven Präferenzen lässt sie kleine Veränderungsschritte ablehnen. Dies kann zur Folge haben, dass charismatische Verführer, die eine völlige Umwälzung hin zu einer besseren Welt versprechen, pragmatischen Reformern, die kleine Veränderungen propagieren, vorgezogen werden. Indessen kann die "Psychologie der Massen" hier nicht thematisiert werden $^{21}$. Nur so viel, um Missverständnisse zu vermeiden: Wunschdenken, das unrealistisch ist und das von Demagogen angeheizt werden kann, ist in keiner Verfassung der Gesellschaft ausgeschlossen. Hier geht es mir darum, dass im Rahmen realistischer, also vosichtig abgeschätzter Alternativen zum Status Quo die Adaptivität der Präferenzen eine, wenn man so will, stark abbremsende Rolle spielen.

Wenn nun die Grundeinstellung der Bürger status- quo-orientiert, also adaptiv ist, bedeutet das Prinzip der Mehrheitsentscheidung, dass eine Überwindung des Status Quo in einer durchpolitisierten Gesellschaft, in der alles zentral entschieden wird, kaum möglich ist. Veränderung kommt in der modernen Gesellschaft von den dezentral aufgebauten Entscheidungssystemen. Das sind insbesondere die Wirtschaft in ihrer marktwirtschaftlich-wettbewerblichen Gestalt, die Wissenschaft in einem Regime der freien Forschung, sowie die Zivilgesellschaft, z.B. in der Form von spontan sich bildenden "NonGovernment-Organisations" (NGO). Dezentrale Entscheidungen werden vom Motiv her nicht im Interesse des Gemeinwohls gefällt. Der Unternehmer, der Wissenschaftler, der zivilgesellschaftliche Akteur unterliegt primär keiner Einzelfall-Kontrolle, ob er auch im Interesse des Gemeinwohls handle. Dezentrale Entscheidungsstrukturen sind geradezu dadurch definiert, dass die in ihnen Entscheidenden von der Gemeinwohlorientierung entlastet sind. Sie können legitimerweise ihren Eigeninteressen nachgehen. Es ist dann Funktion eines geeigneten institutionellen Rahmens (der allmählich durch "Piecemeal Engineering" verbessert werden kann), dafür zu sorgen, dass die EigenwohlOrientierung der dezentral organisierten Entscheidungsträger zugleich dem Gemeinwohl dient. Der Unternehmer, der im Interesse seines eigenen Gewinns den Produktionsablauf rationalisiert, trägt dann zum Gemeinwohl bei, wenn die von ihm freigesetzten Produktionsfaktoren rasch anderweitig wieder Beschäftigung finden und wenn er vom Wettbewerb gezwungen wird, seine Kostensenkung zugleich mit einer Preissenkung zu verbinden. Wenn er Teil

\footnotetext{
${ }^{21}$ Vgl. Le Bon (1895)
} 
eines Wissenschaftssystems ist, in dem tatsächlich Stellen nicht durch Nepotismus, sondern nur durch wissenschaftliche Leistung erlangt werden können, trägt der Wissenschaftler, der im Interesse seiner eigenen Karriere seine ganze Kraft in das Gelingen einer neuen Erkenntnis steckt, zum Gemeinwohl bei. Die Entlastung von der Gemeinwohlorientierung des dezentral Entscheidenden ist die Voraussetzung dafür, dass er so häufig gegen den Status Quo entscheidet. Wäre er direkt dem Gemeinwohl verpflichtet und wäre diese Verpflichtung mit Sanktionen versehen, dann wäre es für ihn am bequemsten, sich für die jeweilige Default-Option zu entscheiden, also für den Status Quo. Denn man wird ihm kaum nachweisen können, dass er damit falsch gehandelt hat. Ausreden, weshalb etwas Neues nicht geht, findet man immer. Sie sind jedenfalls wesentlich leichter zu finden, als der Nachweis, dass ein geplanter Ausbruch aus dem Status Quo auch wirklich dem Gemeinwohl dient. Es ist schwer, Nichthandeln staatlicherseits zu bestrafen, wohingegen es wesentlich leichter ist, falsches Handeln ex post als solches festzustellen und dann zu bestrafen. Die beste Überlebensstrategie in einer Bürokratie ist die Verhinderung von Reformen.

Am Beispiel der Einführung eines neuen Produkts durch einen Unternehmer zeige ich, wie der Präferenz-Konservatismus der Bürgermehrheit im Interesse des Fortschritts unterlaufen werden kann. Ich spreche hier vom PräferenzUnternehmer. Wenn die Präferenzen der Konsumenten beeinflussbar, insbesondere adaptiv sind, dann besteht die Möglichkeit seitens eines Innovators, mit einem Produkt erfolgreich im Markt zu sein, selbst wenn dessen Akzeptanz vor seiner Einführung nicht ausreichen würde, um die Kosten der Entwicklung, Produkteinführung und Herstellung zu decken. Denn die Präferenzen der Bürger können sich gerade durch die Präsenz des Produkts im Markt so verändern, dass nach Markteinführung die Nachfrage wesentlich höher ist als sie bei den ex-ante-Präferenzen gewesen wäre. Gerade diese Präferenzänderung entspricht dem Gedanken der adaptiven Präferenzen, widerspricht aber der Vorstellung fixer Präferenzen. Ein Paradebeispiel für derartige Präferenz-Unternehmer ist Steve Jobs, der mit seinen Produktinnovationen das Unternehmen Apple sehr erfolgreich gemacht hat, obwohl wahrscheinlich ein politischer Entscheidungsprozess in Sachen derartiger Produkte immer eine negativen Ausgang gehabt hätte. 
Grundsätzlich: Demokratie wäre missverstanden, wenn man ein politisches System eines "Primats der Politik" darunter verstünde. Eine Demokratie, in der alle wichtigen Entscheidungen zentralisiert gefällt würden, wäre angesichts der adaptiven Präferenzen zur Stagnation verurteilt. Damit aber würde sie das Wohlfahrtspotential für die Bürger verfehlen, wobei letzteres sich auch bei variablen Präferenzen anhand des Begriffs der Fortschritts-Sequenzen analytisch sauber darstellen lässt. Und deren Nichtzirkularität verdankt sich ja genau wieder den adaptiven Präferenzen. Demokratie ist mit dem Ruf nach Wohlstand somit nur vereinbar, wenn die Wirtschaft und die Wissenschaft dezentral aufgebaut sind und damit gerade von der Gemeinwohl-Orientierung entlastet sind. Sofern eine Mehrheit der Bevölkerung nicht dauerhaft bereit ist, auf Wohlstand zu verzichten, bedarf es der Marktwirtschaft, um die Demokratie zu stabilisieren.

\section{$\underline{\text { G Marktwirtschaft wird durch Demokratie legitimiert. Teil } 1}$}

Demokratie entspricht nicht nur dem Mehrheitsprinzip, sondern auch dem öffentlichen, möglichst sanktionsfreien Diskurs. Die Erwartung oder Hoffnung in der Habermas-Schule ist, dass sich in diesem Diskurs der "eigentümlich zwanglose Zwang des besseren Arguments" durchsetzt. Gleichgültig, ob man diese Erwartung teilt oder nicht teilt, gleichgültig auch, welcher Wahrheitstheorie man anhängt, so ist es jedenfalls ein Kampf oder Wettbewerb der Meinungen, der den öffentlichen Raum auszeichnet. Denn endgültige Wahrheiten gibt es nicht; endgültige Werte gibt es nicht. Daher muss um beides ständig gestritten werden.

Überhaupt herrscht in der Demokratie der politische Wettbewerb. Es ist dies erstens ein Wettbewerb zwischen Personen und Personengruppen wie Parteien. Es ist dies andererseits ein Wettbewerb der Meinungen und ein Wettbewerb der Arten und Weisen, wie die soziale und die natürliche Wirklichkeit erkannt und interpretiert werden. Und es ist schließlich damit auch ein Wettbewerb der Präferenzen.

Demokratie kann nicht verstanden werden, wenn man nicht anerkennt, dass sich die Menschen gegenseitig in ihren Präferenzen beeinflussen. Hier geht es nicht nur um Präferenzen für Waren, die auf dem Markt gekauft werden 
können. Es geht auch um Präferenzen für Ideen, moralische Vorstellungen und ästhetische Ideale. Auch die Präferenzen für bestimmte Waren sind oft Ausdruck der Präferenz für bestimmte Ideale, moralische oder ästhetische Vorstellungen oder für ein bestimmtes Weltverständnis. Am augenscheinlichsten wird dies auf dem Markt für Bücher, Filme oder ganz allgemein für Medien.

Die herkömmliche Welfare Economics hat sich auf ein Denkschema konzentriert, in dem die Präferenzen fest vorgegeben sind. Ihr entsprechend hat auch die Public Choice Literatur ihren Schwerpunkt in Modellen, deren Agenten feste Präferenzen haben. Jedoch, der interpersonelle Einfluss auf die individuellen Präferenzen und Werte ist nicht die Ausnahme, sondern die Regel. Die menschliche Zivilisation basiert ganz wesentlich auf diesen interpersonellen Einflüssen. Man denke nur an die Erziehung der Kinder durch die Eltern.

Ich unterteile die interpersonellen Einflüsse auf Präferenzen in die folgenden Kategorien. Vorab unterscheide ich zwischen dem Beeinflusser (Person B) und dem Beeinflussten (Person A). Wir trennen nun Einflüsse, die vom Beeinflusser B beabsichtigt sind, von solchen, die unbeabsichtigt erfolgen, quasi als Nebenwirkung des Verhaltens des Beeinflussers B. Aus der Sicht des Beeinflussten $A$ unterscheiden wir danach, ob der Einfluss von B dominant ist oder ober er im Wettbewerb mit anderen Einflüssen auf A steht. Im folgenden Schema gebe ich zu jeder der durch diese doppelte Zweiteilung entstehenden vier Klassen von Einflüssen je ein Beispiel an

\begin{tabular}{|lr|l|lr|}
\hline $\begin{array}{l}\text { Beispiele für vier Klassen } \\
\text { von interpersonellen } \\
\text { Einflüssen }\end{array}$ & Einfluss auf A dominant & $\begin{array}{l}\text { Einfluss auf A im } \\
\text { Wettbewerb }\end{array}$ \\
\hline $\begin{array}{l}\text { Einfluss von B } \\
\text { beabsichtigt }\end{array}$ & Eltern erziehen Kinder & $\begin{array}{l}\text { Kommerzielle Werbung } \\
\text { auf Märkten mit } \\
\text { Wettbewerb }\end{array}$ \\
\hline $\begin{array}{l}\text { Einfluss von } \\
\text { unbeabsichtigt }\end{array}$ & B & $\begin{array}{l}\text { Psychische Abhängigkeit } \\
\text { des A von seinem Idol B }\end{array}$ & $\begin{array}{l}\text { Herden-Verhalten; } \\
\text { "Psychologie der } \\
\text { Massen“ d d }\end{array}$ \\
\hline
\end{tabular}


Wie die Beispiele zeigen, gibt es fließende Übergänge. So gibt es neben dem vielleicht zuerst dominanten Einfluss der Eltern auf das Kind in Abhängigkeit von dessen Alter zunehmend konkurrierende Einflüsse. Von diesen sind einige vielleicht "delegierte" Einflüsse, in denen die Eltern andere Personen beauftragen, auf das Kind erzieherischen Einfluss zu nehmen. Andere Einflüsse auf das Kind mögen von der Art sein, dass die Eltern diese Einflüsse am liebsten verhindern würden. Hier entsteht damit eine Art Wettbewerb der Einflüsse. Die Adoleszenz des Kindes mag verstanden werden als die Altersperiode, in der der dominante Einfluss der Eltern abgelöst wird durch einen Wettbewerb zwischen verschiedenen Beeinflussern, also durch einen Wettbewerb zwischen verschiedenen Einflüssen.

Auch zwischen den beabsichtigten und den unbeabsichtigten Einflüssen gibt es einen fließenden Übergang. Eltern wollen ihre Kinder ohne Zweifel beeinflussen. Jedoch üben sie auf Kinder auch einen ganz unbeabsichtigten Einfluss aus. Wenn die Eltern zum Beispiel Raucher sind, so erhöht sich die Wahrscheinlichkeit, dass auch die Kinder Raucher werden, ohne dass dieser Einfluss von den Eltern beabsichtigt gewesen sein muss. Dennoch mögen sich die Eltern dieses Einflusses bewusst sein; er wird dann als Nebenwirkung eigenen Handelns in Kauf genommen. Das ist quasi eine Zwischenlage zwischen beabsichtigtem und nicht beabsichtigtem Einfluss.

Es geht mir letztlich um die Frage, welches Verhalten und damit auch: welche Präferenzen des Bürgers seitens des Staates legitimiert sind. Diese Frage stellt sich bezüglich der Präferenzen überhaupt nicht, wenn man annimmt, dass die Präferenzen fest vorgegeben sind. Wenn man anerkennt, dass es interpersonelle Einflüsse auf die Präferenzen gibt, stellt sich aber diese Legitimationsfrage: Welche Einflüsse auf die Präferenzen anderer Personen sind geeignet, die daraus resultierenden Präferenzen auch gesellschaftlich zu legitimieren?

Gesellschaftliche Legitimierung des Handelns der Bürger ist auch und gerade für eine Gesellschaft freier Bürger erforderlich. Denn dem Staat gehört das Gewaltmonopol. Damit geschieht ohne Widerstand seitens anderer Bürger nur das, was vom Staat geduldet wird, somit aus staatlicher Sicht legitimes Handeln ist. Da aber die Freiheit des Bürgers das Gewaltmonopol voraussetzt, ergibt sich, dass freies Handeln des Bürgers immer nur insoweit besteht, als es sich 
um gesellschaftlich-staatlich legitimes Handeln handelt. Hieraus ergibt sich natürlich die permanente Gefahr für die bürgerliche Freiheit, dass die Mehrheit sich entscheidet, die Handlungsmöglichkeiten des Bürgers übermäßig einzuschränken, also die Freiheit des Bürgers zu missachten. Verfassungsbestimmungen wie die Grundrechte im deutschen Grundgesetz oder die Amendments der US-amerikanischen Verfassung haben primär die Funktion, dieser Gefahr des Freiheitsentzugs per Mehrheitsbeschluss zu wehren.

Wir wollen an der Grundkonzeption einer freiheitlichen Gesellschaft festhalten und uns hierzu auch auf den Ansatz des normativen Individualismus stützen. Das aber bedeutet: wir können zwischenmenschliche Einflüsse auf die Präferenzen keiner umfassenden staatlichen "Qualitätskontrolle" unterwerfen. Es ist andererseits auch problematisch, jedwede Art der Manipulation von Bürgern durch andere Bürger staatlicherseits zuzulassen.

Den Begriff der "Manipulation" können wir in die Nähe der oben diskutierten Klasse der dominanten und absichtsvollen Beeinflussung rücken. Aber auch wenn Bürger $A$ durch Bürger $B$ in diesem Sinne manipuliert wird, braucht das Ergebnis kein schlechtes zu sein. Das hierzu gehörige Beispiel der Erziehung belegt dies. Allerdings unterliegt auch die elterliche Kindererziehung einer gewissen staatlichen Aufsicht - in einem liberalen Staat allerdings einer sehr weitmaschigen. Diese Aufsicht scheint im wohlverstandenen Interesse des Kindes auch angebracht. Auch die in der Moderne allgemein eingeführte Schulpflicht mag eine Form sein, in der man diese Aufsicht auf indirekte Weise ausübt. Auch das Verbot der Kinderarbeit kann aus dieser Sicht gesehen werden.

Es gibt in der Rechtswissenschaft den Begriff der Privatautonomie. Ohne hier in eine umfassende Begriffsbestimmung einzusteigen, kann man einen Kernbestandteil dieses Begriffs wohl in der folgenden Feststellung sehen: ein gesellschaftliches System von Rechtsvorschriften entspricht dem Prinzip der Privatautonomie, wenn seine Architektur der Vermutung gerecht wird, dass der erwachsene Normalbürger selbst am besten weiß, welche seiner Handlungsalternativen seine eigene Wohlfahrt am besten fördert.

Will man dieses Prinzip der Privatautonomie auch dann aufrecht erhalten, wenn man die Universalität der interpersonellen Einflüsse auf Präferenzen und 
Werte als Faktum akzeptiert, dann kommt man zum Wettbewerb der Einflüsse auf die Präferenzen oder auch zum Wettbewerb der Meinungen als einem Kriterium der gesellschaftlichen Legitimierung dieser Einflüsse.

Meine These ist damit diese: Interpersonelle Einflüsse auf Präferenzen sind in der Regel dann kein Hinderungsgrund, die Präferenzen der Bürger als legitimen Maßstab der Wohlfahrt der Bürger anzuerkennen, wenn verschiedene Beeinflusser bei der Einflussnahme auf die Bürger zueinander im Wettbewerb stehen, wenn der Bürger somit keinem Einflussmonopol unterliegt.

Es ist hier nicht der Ort, diese These zu konkretisieren und anschaulich zu machen. Nur so viel sei gesagt: sie steht im Gegensatz oder zumindest in einem Spannungsverhältnis zu der Behauptung, dass der Bürger in der "kapitalistischen" Welt im Grunde keine Möglichkeit des autonomen Handelns habe. Diese Behauptung wird zum Beispiel in der "Frankfurter Schule" der Soziologie und Sozialphilosophie vielfach vertreten. In der Regel besteht in der kapitalistischen Gesellschaft ein Beeinflussungswettbewerb und somit eine "Wahlfreiheit" der Bürger. Dass diese Wahlfreiheit bei der Adoption unterschiedlicher Präferenzen in ihrer konkreten Gestalt immer auch eingeschränkt ist, ist zuzugeben, sollte aber nicht dazu führen, diese nur deshalb ganz zu negieren, weil die Idealvorstellungen des betrachtenden Sozialphilosophen nicht zu den gegenwärtig wählbaren Alternativen gehören. Andererseits sollte "kritische Theorie" im Wettbewerb der Meinungen ernst genommen werden und die dort entwickelte Grundsatzkritik am vorherrschenden System nicht einfach ignoriert werden. Aber auch eine "kritische Theorie" sollte sich umgekehrt mit dem Popperschen Ansatz einer Offenen Gesellschaft und mit der hier behaupteten Problematik der Stagnationstendenz einer überzentralisierten Entscheidungsstruktur auseinander setzen.

Verwandt mit unserer These ist die folgende Aussage: die Kommunikationsstruktur einer deliberativen Demokratie ist Bedingung dafür, dass man die Präferenzen der Bürger als legitimen Maßstab der Wohlfahrt der Bürger anerkennen kann.

In diesem Sinne setzt nicht nur die Demokratie die Marktwirtschaft voraus; umgekehrt setzt bei endogenen Präferenzen und insbesondere 
interpersonellen Einflüssen auf Präferenzen die Legitimität der Marktwirtschaft die deliberative Demokratie voraus.

\section{$\underline{\text { H Marktwirtschaft wird durch Demokratie legitimiert. Teil } 2}$}

Ich kehre nunmehr zum Thema der Kohärenz und daher Stabilität des Piecemeal Engineering zurück. Im Abschnitt $D$ habe ich darauf verwiesen, dass adaptive Präferenzen der Bürger Voraussetzung dafür sind, dass Fortschrittssequenzen immer nicht-zirkulär sind, dass damit der Begriff "Fortschritt" mit einem sinnvollen Inhalt gefüllt werden kann. Die zugrundeliegende axiomatisch-formale Theorie (vgl. den Anhang 1 unten) ist ausgearbeitet worden anhand des Beispiels intra-personeller Rückkopplung zwischen dem Konsum von Waren und den Präferenzen für Waren. Es stellt sich aber heraus, dass die entsprechende formale Theorie allgemeiner ist und zumindest wichtige Formen interpersoneller Präferenzeinflüsse mit umfasst. Gegenwärtig ist nicht abzusehen, wie weit die formale Theorie noch verallgemeinert werden kann.

Die vielleicht wichtigste Form interpersoneller Präferenzeinflüsse ist die der Nachahmung oder Imitation. Sie kann im täglichen Leben ständig beobachtet werden. Wir wollen sie hier nicht im Einzelnen analysieren. Nur so viel hierzu. Wie in Abschnitt $C$ oben dargestellt, ist unser Begriff von Präferenzen derart, dass er alle Freiheitsbereiche der Personen im jeweiligen Status Quo umfasst. Damit wird auch die Nachahmung anderer Personen als Ausdruck der Präferenz der handelnden Person verstanden, es sei denn, das entsprechende Verhalten sei im Rahmen von Reformvorschlägen für die Gesetzgebung schon genauer unter die Lupe genommen worden. Das aber ist im Fall der Nachahmung ganz überwiegend nicht der Fall. Nun ist Nachahmung sicher sehr oft eine der effizientesten Verhaltensweisen in einer Situation unvollkommener Information oder Unsicherheit ${ }^{22}$. Das Kind lernt quasi instinktiv sehr viel durch die Nachahmung der Personen in seiner Umgebung. Sprechen, also den Umgang mit der "Muttersprache", lernt das Kind durch Nachahmung des Sprechens der Mutter. Aber auch reife Persönlichkeiten wissen, dass es zu aufwendig wäre, sich vor jeder Handlung genau zu informieren; und sie

\footnotetext{
${ }^{22}$ Vgl. den Ausspruch von John Maynard Keynes: „Worldly wisdom teaches that it is better for reputation to fail conventionally than to succeed unconventionally." Keynes (1936), Chapter 12.
} 
verlassen sich dann aus Zeitersparnisgründen vielfach auf die Strategie, andere Personen, von denen man bessere Kenntnisse erwartet, nachzuahmen. Sitten und Gebräuche werden durch Nachahmung eingeübt. Gleiches gilt meist für den Kauf neu auf dem Markt erscheinender Güter: der Nachbar kauft es und scheint zufrieden zu sein - also probiert man es selber aus.

Man kann zeigen, dass Nachahmung formal dem intra-personellen Pattern der adaptiven Präferenzen entspricht, sodass die Theoreme 1 und 2 auch auf nachahmendes Verhalten angewendet werden können. Um den Grundgedanken dieser Theorie klar zu machen, verwende ich ein möglichst einfaches Beispiel theoretischer Art. Aber die Theorie ist wesentlich allgemeiner. Im Anhang 2 stelle ich dieses Beispiel dar. Ich stelle hier die allgemeine mathematische Theorie nicht dar, aufgrund derer ich der Auffassung bin, dass sich bei interpersonellen Präferenzeinflüssen, die die Form der Nachahmung annehmen, immer herausstellt, dass Fortschrittssequenzen nicht-zirkulär sind. Ich betone in diesem Zusammenhang, dass das Modell, mit dessen Hilfe ich die Nichtzirkularität von Fortschrittssequenzen auch bei nachahmendem Verhalten nachweise, einer Vorstellung entspricht, dass entweder Wettbewerb zwischen verschiedenen nachzuahmenden Vorbildern besteht oder dem nachzuahmenden Vorbild nicht an einer Manipulation des Nachahmers gelegen ist. Treffen beide Voraussetzungen nicht zu, so kann es Gründe geben, seitens des Staates einzugreifen, obwohl möglicherweise eine Nichtzirkularität von Fortschrittssequenzen vorliegt. Ich habe in einer anderen Arbeit das Thema "kommerzielle Werbung" unter diesem Aspekt einer möglichen Staatsintervention behandelt. Dabei geht es zum Beispiel um solche Fragen wie Suchtgefährdung aufgrund von Werbung ${ }^{23}$ für bestimmte Produkte. Ich behaupte dort, dass diese Staatsintervention "nicht-paternalistisch" sei. Diesen Gedanken kann ich hier nicht vertieft darstellen.

Im vorangehenden Abschnitt hatte ich argumentiert, dass die deliberative Demokratie eine geeignete Plattform dafür ist, dass Präferenzen, die interpersonellen Einflüssen ausgesetzt sind, legitimierbar sind. Dies ist jedoch unter den Vorbehalt der "Kohärenz" des Gesamtsystems zu stellen. Hierfür ist die Nichtzirkularität von Fortschrittssequenzen ein guter Indikator. Insofern ist die Möglichkeit, große Teilmengen von interpersonellen Präferenzeinflüssen

\footnotetext{
${ }^{23}$ Von Weizsäcker (2014b)
} 
formal dem Kalkül zu unterstellen, den wir für adaptive Präferenzen entwickelt haben, ein gutes Anzeichen dafür, dass Kohärenz auch bei interpersonellen Präferenz- und Werteinflüssen gegeben ist.

Diese Nichtzirkularität von Fortschrittssequenzen muss im Grunde auch Habermas unterstellen, wenn er sein Idealbild eines herrschaftsfreien Diskurses propagiert. Der "eigentümlich zwangslose Zwang des besseren Arguments" muss doch bedeuten, dass diesem Diskurs ein "Gedächtnis" anhaftet, der es ermöglicht, auch später Positionen zurückzuweisen, die schon früher einmal durch "bessere" Argumente, also durch argumentativen Fortschritt überwunden worden waren. Ich vermute, dass es einer ähnliche Struktur der Adaptivität der Überzeugungskraft von Argumenten bedarf, damit man auch für den herrschaftsfreien Diskurs eine derartige Nichtzirkularität von Diskursfortschritten nachweisen kann. Nicht nur Karl Popper kann seine "Offene Gesellschaft" nicht mit den "Hans im Glücks" bevölkern; auch Jürgen Habermas muss die argumentativen "Hans im Glücks" in die Schranken verweisen.

Die Frage, die ich in dieser Arbeit nicht weiter behandeln kann, ist die nach der Interdependenz zwischen dem marktwirtschaftlichen System als Voraussetzung der Demokratie und der deliberativen Demokratie als Voraussetzung des marktwirtschaftlichen Systems. Hierzu nur folgendes:

Das marktwirtschaftliche System hat eine Kommunikationsinfrastruktur hervorgebracht, die in seinen Zentralisierungs- und Dezentralisierungswirkungen so nicht vorausgesehen wurde. "Öffentlichkeit" ist durch das Internet etwas anderes geworden als früher. Gleiches gilt für die Marktwirtschaft selbst. Der Ruf nach staatlicher Überwachung solcher Strukturen und Unternehmen wie Google oder Facebook ertönt heute sehr häufig. Sind die Eigengesetzlichkeiten dieser Informationsstrukturen dem deliberativen demokratischen Gemeinwesen abträglich? Oder ist es nur der ausgeprägte Präferenzkonservatismus der Kommentatoren, der sich in diesen Vermutungen äußert? Oder ist es sogar der Fall, dass wir dabei sind, neue Demokratieformen zu entwickeln, die, wenn sie erst einmal etabliert sind, 
gemäß dem Prinzip der adaptiven Präferenzen als den älteren Formen weit überlegen angesehen werden ${ }^{24}$ ?

Auch die in den USA vordringende massive politische Spendenaktivität von reichen Personen, großen Unternehmen oder Religionsgruppen führt zu der Frage, ob es Grenzen der interessengebundenen Beeinflussung auf den politischen Prozess geben sollte.

\section{$\underline{\text { S Schlussbetrachtung }}$}

Die moderne Gesellschaft kann verstanden werden als ein System des Wettbewerbs. Der Wettbewerb findet nicht nur zwischen Unternehmen, Personen und Produktionsverfahren statt, sondern er ist auch ein Wettbewerb der Einflüsse auf die Präferenzen und auf die Werte der Bürger. Den Wettbewerb erster Art rechnen wir der Marktwirtschaft zu. Den Wettbewerb zweiter Art verbinden wir mit dem Gedanken der "deliberativen" Demokratie. Wir können nach dem Vorschlag von Karl Popper uns das Konzept der Offenen Gesellschaft aneignen. Die Offene Gesellschaft muss Kohärenzeigenschaften aufweisen, damit es möglich ist, ein in sich konsistentes Konzept des Fortschritts abzuleiten. Voraussetzung dafür, dass diese Kohärenz vorliegt, ist, dass die endogen bestimmten Präferenzen die Eigenschaft der Adaptivität haben. Diese Eigenschaft ist empirisch vorhanden. Und auch interpersonelle Einflüsse auf Präferenzen, insbesondere die Nachahmung anderer Personen, besitzen Eigenschaften, die den adaptiven Präferenzen ähnlich sind. Insoweit kann die Kohärenz der Offenen Gesellschaft auch bei interpersonellen Präferenzeinflüssen vermutet werden.

So gibt es Hinweise, dass der aufklärerische optimistische Glaube an den Fortschritt auch heute eine Leitlinie für das Nachdenken darüber abgibt, wie das Gesamtwohl am besten gefördert werden kann. Aber, genau weil es einen Wettbewerb der Meinungen und Werte gibt, kann die Debatte über die gute Staats- und Wirtschaftsordnung nie an einem ruhenden Endpunkt ankommen. Die Suche nach besseren Antworten ist Teil einer Offenen Gesellschaft. Auch die Gefahr eines Scheiterns der freiheitlichen Gesellschaft kann nie im Sinne

\footnotetext{
${ }^{24}$ Vgl. zu diesen Fragen Sunstein (2007)
} 
eines mathematischen Beweises ausgeschlossen werden. Es gibt keine endgültigen Wahrheiten oder Werte oder Präferenzen.

\section{Anhang 1: Theoreme 1 und 2}

Hier eine kurze Darstellung der beiden Theoreme zu den adaptiven Präferenzen Vorausgesetzt ist ein Präferenzsystem, das eine Person charakterisiert. Wir bezeichnen es mit dem Buchstaben $\mathcal{P}$. Es besteht aus drei Komponenten: 1 . Ein Objektraum $\mathcal{X}$ bestehend aus Objekten $x$, auf die sich die Präferenzen beziehen. Wir sprechen hier auch von "Warenkörben" $x$ 2. Ein Raum möglicher Präferenzordnungen $\mathcal{Q}$, mit verschiedenen Präferenzordnungen $q$, von denen eine Präferenzordnung zu jedem Zeitpunkt gültig ist. 3. Ein "Bewegungsgesetz" $\dot{q}=f(x ; q)$, das beschreibt, wie sich die Präferenzordnung durch die Zeit verschiebt in Abhängigkeit des tatsächlich konsumierten Warenkorbs $x$ und in Abhängigkeit der jeweils gerade gültigen Präferenzordnung $q$.

Das Präferenzsystem $\mathcal{P}=\{X ; \mathcal{Q} ; f\}$ wird spezifiziert mittels folgender Annahmen:

Annahmen zum Objektraum $x$. Dieser sei eine Teilmenge des $n$-dimensionalen Euklidischen Raums $R^{n}$, zum Beispiel eine kompakte Teilmenge des Orthanten der nichtnegativen Vektoren $x \geq 0$.

Annahmen zum Raum der Präferenzen $\mathcal{Q}$. 1a. Die Präferenzordnungen, die in $\mathcal{Q}$ enthalten sind, sind stetig im üblichen Sinne dieses Wortes (vgl. z.B. Debreu (1959), S. 56). 1b. Ferner gilt Stetigkeit des Übergangs von einer Präferenzordnung in eine andere im folgenden Sinne: Der Ausdruck $y(>; q) x$ soll heissen: bei der Präferenzordnung $q$ wird der Warenkorb $y$ dem Warenkorb $x$ vorgezogen. Es wird angenommen, dass jeder Vektor $x$ aus $X$ eine Präferenzordnung $\rho(x)$ aus $\mathcal{Q}$ induziert. Damit ist intuitiv gemeint: wenn der Warenkorb $x$ konstant durch die Zeit bleibt, dann konvergiert die Präferenzordnung gegen die induzierte Präferenzordnung $\rho(x)$. Stetigkeit des Übergangs von einer Präferenzordnung zur anderen soll nun heißen: Gilt $y(>; \rho(z)) x$, dann gibt es eine Umgebung $\mathcal{U}(z)$ von $z$ derart, dass für $w \in \mathcal{U}(z)$ ebenfalls gilt $y(>; \rho(w)) x$. 2. Ferner sollen alle Präferenzordnungen die Eigenschaft der Nichtsättigung (Non-Satiation) in diesem Sinne haben: ist $y$ in 
jeder Komponente größer als $x$, dann gilt für alle $q \in \mathcal{Q}$ die Beziehung $y(>$ ;q) $x$. 3. Neben der Stetigkeit und Nichtsättigung sollen die Präferenzordnungen noch eine dritte Eigenschaft haben, die ich Regularität nenne. Im Fall, dass es nur zwei Güter gibt (also der zweidimensionale Euklidische Raum), bedeutet Regularität, dass sich zwei Indifferenzkurven zweier Präferenzordnungen, die sich in einem Warenkorb $x$ schneiden, sich nirgendwo sonst noch einmal schneiden ("Single Crossing"- Eigenschaft). Im Fall des mehrdimensionalen Güterraums soll eine entsprechende Eigenschaft gelten, die ich "extended regularity" nenne.

Annahmen zum Bewegungsgesetz der Präferenzen. Hier unterscheide ich zwei Modelle: 1. das "Class Room Model" und 2. das "Real World Model". Im ClassRoom Model arbeite ich mit diskreten Zeitperioden, die ich "Jahre" nenne. Hier nehme ich an, dass die Prägung der Präferenzen durch den faktischen Konsum genau ein Jahr dauert, sodass die Päferenzen, die im Jahr t gelten, die Präferenzen sind, die vom Warenkorb des Vorjahrs t-1 induziert werden. Also $q(t)=\rho(x(t-1))$. Im Text habe ich die Fortschrittssequenz im Rahmen des Class Room Modells definiert. Im Real World Model nehme ich stetige Zeit an, was ja der tatsächlichen Zeit entspricht. Das Bewegungsgesetz der Präferenzen ist dann eine Differentialgleichung durch die Zeit. In diesem Fall muss der Begriff der Fortschrittssequenz etwas raffinierter definiert werden. In diesem Modell ist auch der Begriff "induzierte Präferenzen" nur klar definiert, wenn man den Präferenzenraum als topologischen Raum auffasst, der mit einer Topologie versehen ist. Das bringt keine grundsätzliche Schwierigkeiten, da wir mit stetigen Präferenzen im herkömmlichen Sinne dieses Wortes arbeiten, die uns auch stetige Nutzenfunktionen ermöglichen. Wir können den Raum $\mathcal{X}$ der Warenkörbe auf eine kompakte Teilmenge des n-dimensionalen Euklidischen Raums beschränken. Dann sind auf dieser kompakten Menge alle stetigen Nutzenfunktionen nach dem Stone-Weierstrass-Theorem beliebig genau durch Polynome mit reellwertigen Koeffizienten approximierbar. Dann kann die Topologie des Präferenzenraums die natürliche Topologie des Euklidischen Raums der Polynomkoeffizienten sein, sofern man sich auf Approximationen beschränkt, deren Polynome einen bestimmten Höchstgrad nicht überschreiten. Man kann dann die Stetigkeit des Präferenzübergangs als stetig in dem Sinne verstehen: ist $y(>; q) x$, dann gibt es eine Umgebung $U(q)$ von $q$, 
sodass für $r \in \mathcal{U}(q)$ ebenfalls gilt: $y(>; r) x$. Die Definition der adaptiven Präferenzen bleibt dieselbe wie im Class-Room-Model.

Adaptive Präferenzen wurden schon im Text ansatzweise definiert. Sie liegen dann vor, wenn 1 . aus $y(>; \rho(x)) x$ folgt, dass auch gilt: $y(>; \rho(y)) y$ und wenn 2. aus $y(=; \rho(x)) x$ folgt, dass auch gilt: entweder $y(>; \rho(y)) x$ oder $y(=; \rho(y) y$.

Eine Fortschrittssequenz ist im Class-Room-Model so definiert: es handelt sich um eine endliche Sequenz von Jahreswarenkörben $x^{0}, x^{1}, x^{2}, \ldots . x^{T}$ derart, dass gilt: $x^{0}\left(<; \rho\left(x^{0}\right)\right) x^{1}\left(<; \rho\left(x^{1}\right)\right) x^{2} \ldots \ldots \ldots x^{T-1}\left(<; \rho\left(x^{T-1}\right)\right) x^{T}$.

Theorem 1: Gelten die genannten Annahmen 1a, 1b, 2 und 3 über das Präferenzensystem, dann folgt aus der zusätzlichen Annahme adaptiver Präferenzen, dass alle Fortschrittssequenzen nicht-zirkulär sind.

Dieses Theorem gilt für beide Modelle, das Class-Room-Model und das RealWorld-Model. Der Beweis ist relativ aufwendig, sofern der Euklidische Güterraum eine Dimension größer als 2 hat. Für den Zwei-Güter-Fall und das Class-Room-Model ist der Beweis recht durchsichtig. $\mathrm{Er}$ läuft als Widerspruchsbeweis per vollständiger Induktion bezüglich der Länge der Fortschrittssequenzen. Vorab kann man für zirkuläre Fortschrittssequenzen im Rahmen eines Widerspruchsbeweises den Fall ausschließen, dass es einen Schritt $t$ in der Sequenz gibt, bei dem beide Güter zunehmen: denn, wäre $x^{t}<x^{t+1}$, dann würde wegen Non-Satiation auch gelten $x^{t-1}\left(<; \rho\left(x^{t-1}\right)\right) x^{t+1}$. Dann hätte man durch Eliminierung von $x^{t}$ eine um ein Jahr verkürzte Fortschrittssequenz, die per Induktionsanahme nicht zirkulär ist. Also kann man nutzen, dass jede zirkuläre Fortschrittssequenz (für die also gilt: $x^{T}=x^{0}$ ) irgendwo ihre Richtung von "nach links oben" auf "nach rechts unten" (oder von "rechts unten" nach "links oben") ändert, also einen "Haken" schlägt. Dann kann man zeigen, dass bei diesem "Haken", wenn der im Jahr $t$ stattfindet, auch gilt: $x^{t-1}\left(<; \rho\left(x^{t-1}\right)\right) x^{t+1}$, sofern die Präferenzen adaptiv sind. Damit kann man $x^{t}$ aus der Fortschrittsequenz eliminieren und hat somit bei gleich gebliebenem Anfangs- und Endkorb eine Fortschrittssequenz, die um ein Jahr verkürzt ist, was aber nach Induktionsannahme impliziert, dass der Endkorb nicht gleich dem Anfangskorb ist, im Widerspruch zu der Annahme, dass die Fortschrittssequenz zirkulär sei. Die Verankerung der 
Induktionsannahme für $T=2$ folgt unmittelbar aus der Definition adaptiver Präferenzen, denn wäre $x^{2}=x^{0}$, dann hätten wir $x^{1}\left(>; \rho\left(x^{0}\right)\right) x^{0}$ und $x^{0}\left(>; \rho\left(x^{1}\right)\right) x^{1}$, im Widerspruch zur Annahme adaptiver Präferenzen.

Ein Beweis des allgemeinen Theorems 1 ist enthalten in von Weizsäcker (2013)

Theorem 2 ist allgemeiner als ich es hier darstelle. Hier nun:

Theorem 2 (einfache Form): Sind in einem Präferenzsystem $\mathcal{P}=\{\mathcal{X} ; \mathcal{Q} ; f\}$ die Annahmen 1a, 1b und 2 bezüglich der Präferenzen erfüllt (Stetigkeit und Nonsatiation) und sind in ihm alle Fortschrittssequenzen nicht-zirkulär, dann sind die Präferenzen dieses Systems adaptiv.

Beweis der einfachen Form für den Fall des Class-Room-Model: Wären die Präferenzen nicht adaptiv, so würden wir entweder einen Fall finden, in dem gilt: $y(>; \rho(x)) x$, jedoch $x(\geq ; \rho(y)) y$ oder einen Fall, in dem gilt $y(=$ $; \rho(x)) x$, jedoch $x(>; \rho(y)) y$. Im ersten Fall gäbe es einen Vektor $\varepsilon e>0$ mit einer positiven reellen Zahl $\varepsilon$ und dem aus lauter Einsen bestehenden Vektor $e$ derart, dass gilt $y(>; \rho(x+\varepsilon e))(x+\epsilon e)$ und $(x+\varepsilon e)(>; \rho(y)) y$. Dies deshalb, weil die Präferenzen stetig sind, die Präferenzübergänge $\rho(x)$ stetig sind und wegen der Annahme der Nichtsättigung. Dann aber haben wir einen zirkulären Fortschrittspfad: $x+\varepsilon e ; y ; x+\varepsilon e$, was die Annahme des Theorems verletzt. Im zweiten Fall müssen wir nur die Rollen von $x$ und $y$ vertauschen und bekommen das gleiche Resultat. QED.

Der Beweis einer allgemeineren Form des Theorems 2 ist enthalten in von Weizsäcker (2013).

Anhang 2. Ein einfaches Beispiel für die formale Äquivalenz von nachahmenden und adaptiven Präferenzen.

Wir stellen uns zwei Personen vor, die in einer Zwei-Güter-Ökonomie konsumieren. Person A ist der Nachahmer, Person B ist die Person, die von Person A nachgeahmt wird. Es gibt somit einen inter-personellen PräferenzEinfluss von Person B auf Person A. Beide Personen sind Teil einer größeren Volkswirtschaft, deren Details wir aber für unsere Zwecke nicht in den Blick nehmen müssen. Die Feststellung einer Einbettung in eine größere 
Personengruppe erfolgt nur deshalb, weil ich damit begründen will, dass Person B am Verhalten und damit an den Präferenzen von Person A gar nicht interessiert ist. Für Person $B$ ist ihr Einfluss auf das Konsumverhalten von Person A nur eine irrelevante Nebenwirkung eigenen Verhaltens. Für Person A ist hingegen das Verhalten von Person B von Bedeutung, da sie ja ihr Verhalten zum Teil an dem von Person B ausrichtet, und zwar nachahmend. Vereinfachend unterstellen wir, dass Person B fixe Präferenzen hat.

Wir unterstellen nun weiterhin, dass sich die Nachfrage nach den beiden Gütern bei beiden Personen an den Marktpreisen der beiden Güter und an den jeweiligen Budgets ausrichtet. Im weiteren Verlauf halten wir zwecks einfacherer Darstellung das Budget von Person A konstant, sodass Nachfrageänderungen bei Person $A$ nur auf Preisänderungen und auf den veränderten Konsum von Person B zurückgeführt werden. Die Nachfrage von Person B hängt von ihrem Budget und von den Marktpreisen ab. Schließlich unterstellen wir, dass die Nachfragefunktionen der beiden Personen invertierbar sind: das bedeutet, dass bei gegebenen Präferenzen und gegebenem Budget jedem nachgefragten Warenkorb genau ein Preisvektor entspricht, der diesen Warenkorb als Nachfrage auslöst.

Genauer definieren wir die von Person B beeinflussten Präferenzen von Person A wie folgt. Wir betrachten die den verschiedenen Preisverhältnissen $p=\frac{p_{1}}{p_{2}}$ entsprechenden Engel-Kurven der Person B. Wir geben jeder Engel-Kurve eine "Hausnummer" $s$; ohne Verlust an Allgemeinheit können wir die Hausnummer so wählen, dass gilt $s=p$. Wir nehmen nunmehr an, dass die Präferenzen von Person A bestimmt werden durch den Konsum von Person B in der Weise, dass sie allein von dem Wert von $s$ abhängen, der dem nachgefragten Warenkorb von Person B entspricht. Es seien $q$ die Präferenzen von A. Dann gilt also $q=q(s)$. Wir betrachten nun die Nachfragefunktion von Person A. Diese ist im Allgemeinen $x=f\left(p_{1}, p_{2} ; q(s)\right)$. Und die inverse Nachfragefunktion von $\mathrm{A}$ lautet $\pi \equiv\left(p_{1}, p_{2}\right)=g(x ; q(s))$. Da Person A die Person B nachahmt, können wir annehmen, dass gilt: je größer der Wert von $s$ ist, desto kleiner ist bei gegebenen Preisen die Nachfrage von Person A nach Gut 1 und desto größer ist die bei gegebenen Preisen die Nachfrage nach Gut 2.

Nun definieren wir die "induzierten" Präferenzen des Warenkorbs $x$ von Person A. Wir haben die invertierte Nachfragefunktion $\pi=g(x ; q(s))$. Da aber $s=p$ 
auch durch $\pi$ bestimmt wird, können wir schreiben $q=q(\pi)$ und damit $\pi=g(x ; q(\pi))$. Für vorgegebenes $x$ ist dies eine Gleichung für $\pi$. Diese ist eindeutig lösbar, wenn die Präferenzen von A nur von $s$ abhängen und bei Person B beide Güter "normale" Güter sind, sie also mit steigendem Einkommen bei gleich bleibenden Preisen vermehrt nachgefragt werden. Somit können wir schreiben $\pi=G(x)$ und wir definieren die von $x$ induzierten Präferenzen als $\rho(x)=q(G(x))$.

Nunmehr betrachten wir zwei Warenkörbe $x$ und $y$ von Person A derart, dass gilt $y(>; \rho(x)) x$. Wir wollen nun zeigen, dass dann auch gilt $y(>; \rho(y)) x$. Ist $y$ in beiden Komponenten größer als $x$, dann folgt $y(>; \rho(y)) x$ aus der Annahme der "Non-Satiation", die wir für Theorem 1 und 2 ohnehin machen. Also können wir uns auf den Fall konzentrieren, dass $y$ in einer Komponente kleiner als $x$ ist. Wir betrachten den Fall, dass dass gilt $y_{1}>x_{1}$ und $y_{2}<x_{2}$. Nun muss bezüglich des Preisverhältnisses offensichtlich gelten $p(y ; \rho(x))<$ $p(x ; \rho(x))$. Daher ist der Wert von $s$ bei $y$ kleiner als bei $x$. Das aber bedeutet wegen des nachahmenden Verhaltens von A, dass die Indifferenzkurve durch $y$, die den von $y$ induzierten Präferenzen entspricht, an der Stelle $y$ steiler verläuft als die Indifferenzkurve durch $y$, die den von $x$ induzierten Präferenzen entspricht. Wie die Graphik zeigt, folgt daraus, dass auch gilt $y(>; \rho(y)) x$. Entsprechend läuft der Beweis für den Fall $y_{1}<x_{1}$ und $y_{2}>x_{2}$. Das war zu beweisen, um die Eigenschaft adaptiver Präferenzen auch für die Verhaltensimitation nachzuweisen. Damit können wir auf das interpersonelle Präferenzverhältnis zwischen Personen A und B ebenfalls Theoreme 1 und 2 anwenden. 


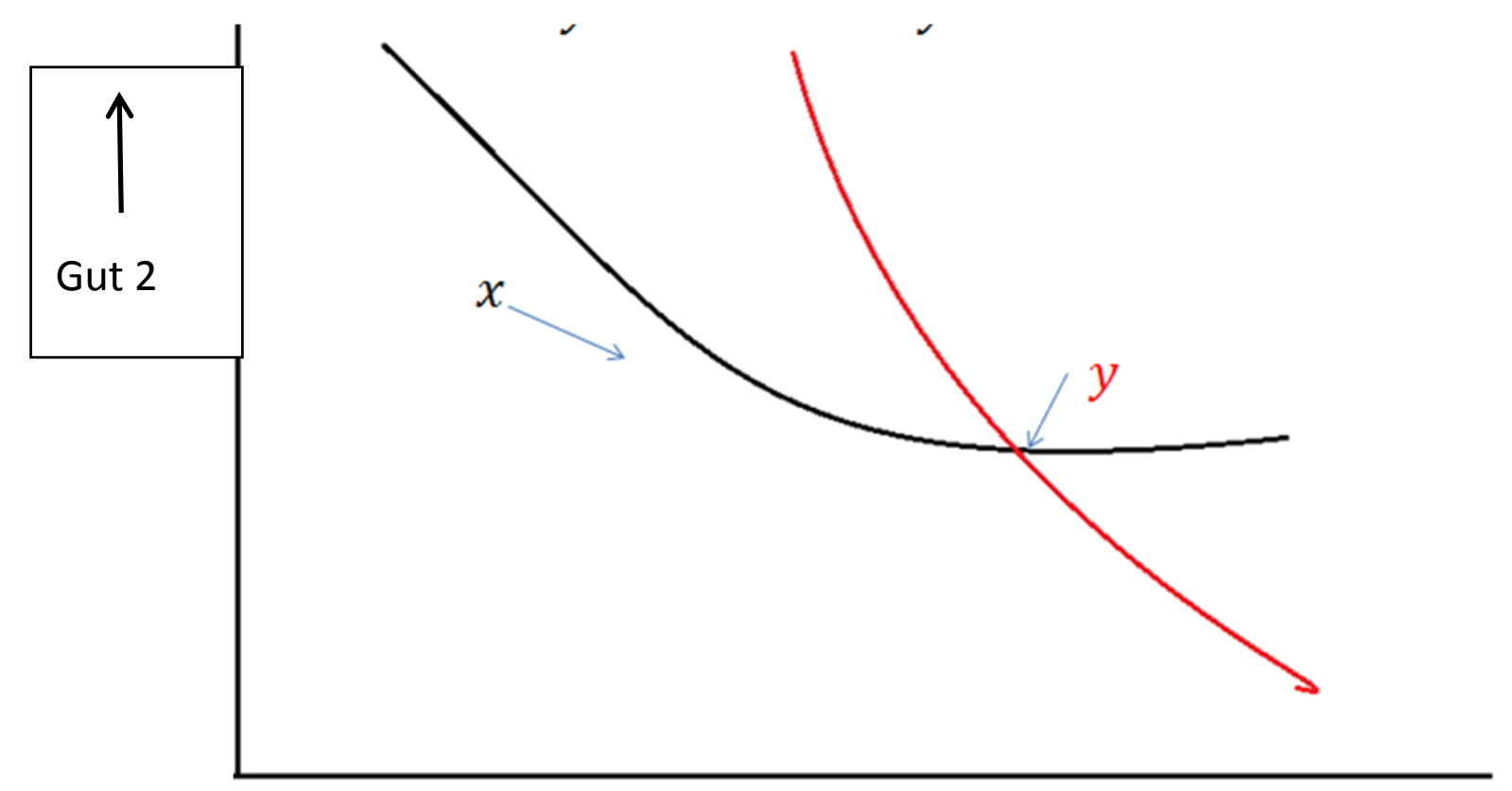

Gut 1

\section{$\underline{\text { Literatur }}$}

Debreu (1959), Gerard Debreu, Theory of Value, New York, 1959

Habermas (1981), Jürgen Habermas, Theorie des kommunikativen Handelns; zwei Bände, Frankfurt, 1981

Habermas (1992), Jürgen Habermas, Faktizität und Geltung, Frankfurt 1992

Hayek(1944), Friedrich August von Hayek, The Road to Serfdom, London 1944

Hayek (1973), Friedrich August von Hayek, Die Anmaßung von Wissen, Ordo 1973

Hirschman (1967), Albert O. Hirschman, Development Projects Observed, Washington DC, 1967

Keynes (1936), John Maynard Keynes, The General Theory of Employment, Interest and Money, London 1936 
Keynes (1939); John Maynard Keynes, Preface to the French Edition of the General Theory, quoted from The Collected Writings of John Maynard Keynes, Vol VII, The General Theory of Employment, Interest and Money, p. XXXIV, London 1973

Kornai (1992), Janos Kornai, The Socialist System: The Political Economy of Communism, Oxford 1992

Lawson et al (2010), Robert Lawson and Jeff R. Clark, Examining the HayekFriedman Hypothesis on Economic and Political Freedom, Journal of Economic Behavior and Organization 74, 230-239

Le Bon (1895), Gustave Le Bon, Psychologie des Foules, Paris 1895

Polanyi (1944), Karl Polanyi, The Great Transformation, New York 1944

Popper (1945), Karl Popper, The Open Society and its Enemies, zwei Bände, London 1945

Simon (1962), Herbert Simon, The Architecture of Complexity, Proceedings of the American Philosophical Society, Vol 106, No 6, 1962, S. 467-482

Stigler, George and Gary Becker (1977), De Gustibus Non Est Disputandum, American Economic Review, 1977, S. 76-90

Sugden (2004), Robert Sugden, The Opportunity Criterion: Consumer Sovereignty Without the Assumption of Coherent Preferences, American Economic Review, 94, 1014-1033

Sunstein (2007), Cass, Republic.com 2.0, revised edition, Princeton 2007, 251 Seiten

Von Weizsäcker (2003), Carl Christian von Weizsäcker, Logik der Globalisierung, 3. Auflage, Göttingen 2003, 173 Seiten

Von Weizsäcker (2013), Carl Christian von Weizsäcker, Freedom, Wealth and Adaptive Preferences, Manuskript 2013, 123 Seiten; abrufbar von meiner Homepage (http://www.coll.mpg.de/team/page/carl-christian von-weizsaecker)

Von Weizsäcker (2014a), Carl Christian von Weizsäcker, Adaptive Präferenzen und die Legitimierung dezentraler Entscheidungsstrukuren, Manuskript, im 
Erscheinen, abrufbar von meiner Homepage (http://www.coll.mpg.de/team/page/carlchristian von-weizsaecker)

Von Weizsäcker (2014b), Carl Christian, Konsumentensouveränität und beeinflussbare Präferenzen. Ist Laisser Faire bei Werbung das Richtige? Manuskript 2014, im Erscheinen, abrufbar von meiner Homepage (http://www.coll.mpg.de/team/page/carl-christian von-weizsaecker) . 